Comment. Math. Helv. 74 (1999) 201-237

(C) 1999 Birkhäuser Verlag, Basel

0010-2571/99/020201-37 \$1.50+0.20/0

Commentarii Mathematici Helvetici

\title{
Compactification of moduli space of harmonic mappings
}

Jingyi Chen and Gang Tian

\begin{abstract}
We introduce the notion of harmonic nodal maps from the stratified Riemann surfaces into any compact Riemannian manifolds and prove that the space of the energy minimizing nodal maps is sequentially compact. We also give an existence result for the energy minimizing nodal maps. As an application, we obtain a general existence theorem for minimal surfaces with arbitrary genus in any compact Riemannian manifolds
\end{abstract}

Mathematics Subject Classification (1991). 53C42, 58E20.

Keywords. Compactness, harmonic maps, stratified Riemann surface.

\section{Introduction}

In many cases, a sequence of harmonic maps contains a subsequence which converges to a limit map. The limit map is a union of smooth harmonic maps. However, it is often difficult to keep track of how topology changes during the limiting process. On the other hand, we do have satisfactory compactness results in certain cases, for example, Gromov's compactness theorem for pseudoholomorphic curves and for perturbed Cauchy-Riemann equations ([Gr], [PW], [RT], [Ye]). In this paper, we make a few new observations on compactness for 2-dimensional harmonic maps and derive some consequences.

To motivate our discussion, let us mention a few well-known examples. First, J. Eells and J.C. Wood ([EW]) show that there are no harmonic maps of degree 1 or -1 from the 2 -torus $\mathbb{T}^{2}$ to the 2 -sphere $\mathbb{S}^{2}$. Second, A. Futaki ([A]) proves that an energy minimizing map from $\mathbb{S}^{2}$ into a Hirzebruch surface $M$ in the class $\alpha+\beta$, where $\alpha$ and $\beta$ are two generators of $\pi_{2}(M)=\mathbb{Z} \oplus \mathbb{Z}$, has to splits into two spheres representing $\alpha$ and $\beta$ respectively. Finally, if one starts from a long cylinder in $\mathbb{R}^{3}$ and deforms it continuously to minimize area of surfaces spanning the two boundary circles, then one gets two flat discs joined by a line.

Let $\Sigma$ be a stable curve of genus $g$ in the sense of Deligne-Mumford, possibly

The first author is supported by a NSF postdoctoral fellowship and the second author is supported partially by NSF grants. 
with nodes as singularities. Recall $\Sigma$ is obtained by collapsing finitely many circles on a smooth compact Riemann surface of genus $g$ to points and requiring any component which is homeomorphic to $\mathbb{S}^{2}$ touches at least three other components. Let $M$ be a smooth compact Riemannian manifold with a Riemannian metric $h$. As usual, the moduli space of all such $\Sigma$ is denoted by $\overline{\mathcal{M}}_{g}$, which is compact. The open subset of $\overline{\mathcal{M}}_{g}$ consisting of all smooth curves is denoted by $\mathcal{M}_{g}$. A stratified Riemann surface $T$ associated to $\Sigma$ is defined to be a union of smooth compact Riemann surfaces $\Sigma_{1}, \ldots, \Sigma_{k}$ and line segments $l_{1}, \ldots, l_{p}$ of unit length such that (i) $\Sigma_{i} \cap \Sigma_{j}=\emptyset$ and $l_{i} \cap l_{j}=\emptyset$ for any $i \neq j$; (ii) each $l_{i}$ intersects $\cup_{j=1}^{k} \Sigma_{j}$ transversely at two points and $T$ is connected; (iii) if $l_{j}$ 's are removed and the end points of $l_{j}$ are identified, we obtain a surface $\Sigma^{\prime}$ possibly with nodes as singularities. Moreover, $\Sigma^{\prime}=\cup_{i=1}^{k} \Sigma_{i}$ is the union of components in $\Sigma$ and some 2-spheres. Each $\Sigma_{i}$ is called a 2-dimensional component of $T$ while $l_{j}$ a 1-dimensional component of $T$. Also, if $\Sigma_{i}$ is a component of $\Sigma$, we call it a principal component of $T$, otherwise, $\Sigma_{i}$ is a bubbling component of $T$. Note that all bubbling components are 2 -spheres, but some principal components may be also 2 -spheres. If there are at most two lines connecting a 2-sphere with other components, we say the 2 -sphere is an unstable component of $T$. A conformal structure on $T$ is given by assigning a conformal structure on each 2-dimensional component. A smooth metric $\mu$ on $T$ consists of smooth metrics $\mu_{i}$ on $\Sigma_{i}$ and the standard metric $d s_{j}$ on $l_{j}$. The regular part $\operatorname{Reg}(T)$ is $T \backslash \cup_{i=1}^{p} l_{i}$. Note that $\operatorname{Reg}(T)$ is the disjoint union of punctured Riemann surfaces. Let $\chi(\operatorname{Reg}(T))$ be the Euler characteristic class of $\operatorname{Reg}(T)$. The genus $g(T)$ of $T$ is given by

$$
g(T)=1-\frac{1}{2} \chi(\operatorname{Reg}(T)) .
$$

For example, take two 2 -spheres $S_{1}, S_{2}$. (a) if $T=\left(S_{1}, S_{2} ; l\right)$ with $l$ joining $S_{1}, S_{2}$, then $g(T)=0$; (b) if $T=\left(S_{1}, S_{2} ; l_{1}, l_{2}\right)$ with each of $l_{1}, l_{2}$ joining $S_{1}, S_{2}$, then $g(T)=1$. We are interested in maps $f$ from $T$ to $M$ satisfying: (1) $f$ is smooth on each $\Sigma_{j}$ and each $l_{i} ;(2) f$ is Lipschitz on $T$. The set of all such maps is denoted by $C^{\infty}(T, M)$. We call a map $f \in C^{\infty}(T, M)$ harmonic nodal map if (i) it is harmonic on each surface $\left(\Sigma_{j}, \mu_{j}\right)$ and on each line $l_{i}$; (ii) if $f$ is constant on some bubbling component $\Sigma_{i}$, then $\Sigma_{i}$ intersects with at least three 1-dimensional components of $T$. The requirement on 1-dimensional components in (i) simply means that $f$ maps the interval $[0,1]$ into a geodesic in $M$. The energy $E(f)$ of $f \in C^{\infty}(T, M)$ is defined to be

$$
E(f)=\sum_{j=1}^{k} \int_{\Sigma_{j}}|\nabla f|_{\mu_{j}}^{2} d \mu_{j} .
$$

Note that (ii) implies that the automorphism group of $T$ is finite.

We have the following compactness theorem for harmonic nodal mappings.

Theorem 1.1. Let $M$ be a compact Riemannian manifold without boundary. Let $T_{n}$ be a stratified Riemann surfaces associated to a stable curve $\Sigma_{n} \in \overline{\mathcal{M}}_{g}$ of the 
same genus for $n=1, \ldots, \infty$. Assume that $\Sigma_{n}$ converges to some stable curve $\Sigma_{\infty}$ in $\overline{\mathcal{M}}_{g}$. Suppose that $\left\{u_{n}\right\}$ is a sequence of energy minimizing harmonic nodal maps from $T_{n}$ to $M$ in the same homotopy class whose energies are uniformly bounded above. Then a subsequence of $\left\{u_{n}\right\}$ converges to an energy minimizing harmonic map from $T_{\infty}$ to $M$, where $T_{\infty}$ is a stratified Riemann surface associated to $\Sigma_{\infty} \in \overline{\mathcal{M}}_{g}$. Further, $u_{n}\left(T_{n}\right)$ converges to $u_{\infty}\left(T_{\infty}\right)$ continuously in the Hausdorff distance. The geodesics from the unstable components are trivial.

We point out that since the total energy of $u_{n}$ is uniformly bounded above, there are at most finitely many extra $\mathbb{S}^{2}$ 's, i.e., the bubbles in $T_{\infty}$. If $g_{n}, g_{\infty}$ are the metrics compatible with conformal structures on $\Sigma_{n}, \Sigma_{\infty}$ respectively (i.e. Kähler metrics with respect to the conformal structures $\left.c_{n}, c_{\infty}\right)$, then that $\Sigma_{n}$ converges to $\Sigma_{\infty}$, means that $g_{n} \rightarrow g_{\infty}$ uniformly on every compact subset away from the singular set of $\Sigma_{\infty}$ and we say the conformal structures $c_{n}$ converge to the conformal structure $c_{\infty}$. In a special case that $T_{n}=\Sigma$ is a fixed Riemann surface, the result in Theorem 1.1 was already obtained by Parker in $[\mathrm{P}]$.

The existence of harmonic maps in given homotopy class has been a central problem in geometric analysis. The important work of Sacks-Uhlenbeck [SU1] asserts the existence of a non-constant harmonic map from $\mathbb{S}^{2}$ into a compact Riemannian manifold $N$ with non-contractible universal cover. Sacks-Uhlenbeck [SU2] and Schoen-Yau [SU] proved independently the following beautiful result on incompressible minimal surfaces: if $\phi: \pi_{1}(\Sigma) \rightarrow \pi_{1}(M)$ is an injective homomorphism, then there exists a branched minimal immersion from $\Sigma$ to $M$ which minimizes area among maps in the same conjugacy class of $\phi$. Note that this does not say that each homotopy class of maps from $\Sigma\left(\right.$ even $\left.\mathbb{S}^{2}\right)$ to $N$ contains a harmonic representative. If allowing degeneration of conformal structure on domain surface $\Sigma$, one can obtain the general existence result (see Theorem 1.2).

In proving the existence theorem in [SU1], Sacks and Uhlenbeck used the minimizers of perturbed energy and then take their limit. The limit may not be necessarily a single smooth harmonic map. It is often a union of several harmonic maps, which may not be connected. One new result of this paper is to provide a complete understanding of the behavior of the minimizing sequence along the "necks" which connect those harmonic components. In particular, we provide detailed analysis on convergence of critical points of Sacks-Uhlenbeck's functional. As a simple corollary, we deduce the existence of harmonic nodal map with given genus in each homotopy class.

Theorem 1.2. Let $M$ be a compact Riemannian manifold without boundary and $T$ a stratified Riemann surface associated to $\Sigma$ in $\overline{\mathcal{M}}_{g}$. For any homotopy class $\left[u_{0}\right] \in[T, M]$ defined by a map $u_{0} \in C^{\infty}(T, M)$, there exists an energy minimizing harmonic map u from $T^{\prime}$, a stratified Riemann surface associated to $\Sigma$ in $\overline{\mathcal{M}}_{g}$, to $M$ in the homotopy class $\left[u_{0}\right]$. Moreover, $u$ is non-constant on the bubbles and maps the lines in $T^{\prime}$ to geodesics with finite length in $M$. In fact, $\left(u, T^{\prime}\right)$ is the 
limit of a sequence of minimizers of the perturbed energies in [SU1].

One consequence of Theorem 1.1 and Theorem 1.2 is:

Theorem 1.3. For any fixed genus $g$ and homotopy class a, there is a minimizing harmonic nodal map into $M$, which is also conformal.

This harmonic nodal map actually parameterizes a generalized minimal surface which minimizes the area among surfaces with genus $g$ and homotopy class $a$ in $M$. In particular, any immersed surface $\Sigma$ in $M$ can be deformed to $\tilde{\Sigma}$, a finite union of compact admissible (see Section 4) minimal surfaces and geodesics of finite length, such that $\Sigma$ and $\tilde{\Sigma}$ have the same genus.

Other possible applications include developing the Morse theory on the space of maps from a Riemann surface via the energy functional. Our theorems here indicate that one can achieve certain compactness by partially compactifying the moduli space of maps.

Large portion of this paper consists of asymptotic analysis near singularities arising either from bubbling off of harmonic maps or from degeneration of conformal structure on domain. Applications of asymptotic analysis to geometric problems were given by Almgren-Allard [AA], Cheeger-Tian [CT], Schoen-Uhlenbeck [ScU], Simon [S1], [S2] and other people in various situations. The second author of the present paper observed the possible collapsing of harmonic maps to geodesics in a unpublished note. Also, there are related important works on singularities of harmonic maps and maps with $L^{2}$-bounded tension fields, notably by Jost, Qing, Wang ([J1], [Q], [DT], [QT], [W]), etc.

We prove the compactness theorem for minimizing harmonic nodal mappings in Section 2 and the existence of harmonic maps in any given homotopy class in Section 3. Then in Section 4, we apply the techniques and results developed in the previous two sections to study the existence of minimal surfaces in given homotopy classes. The construction of minimal surfaces requires two minimizing processes. In the given homotopy class, first find minimizing harmonic nodal maps for the given conformal structures; then minimize the energy among conformal structures. The existence of minimal surfaces has been extensively studied by many people for a very long time and many important results have been obtained (see [Do], $[\mathrm{Gu}],[\mathrm{Hi}],[\mathrm{J} 2],[\mathrm{Mo}],[\mathrm{MY}],[\mathrm{MSY} 1,2],[\mathrm{Os}],[\mathrm{SU} 1],[\mathrm{SU} 2],[\mathrm{SY}],[\mathrm{TT}]$ for example).

We would like to thank M. Struwe for his interests in this work and point out some errors in the early version of this paper. We are grateful to the referee for useful and detailed comments. 


\section{Compactness of minimizing harmonic nodal maps}

In this section, our main goal is to prove Theorem 1.1.

Let $c_{n}$ be the conformal structure with respect to which the map $u_{n}$ is harmonic. By the compactness of $\Sigma$, there exists a subsequence of $\left\{c_{n}\right\}$, which is still denoted by $\left\{c_{n}\right\}$, converges to a conformal structure $c_{\infty}$ (refer to the previous section for definition). $c_{\infty}$ may have nodes as singularities. In each conformal class $c_{n}$, there exists a metric $g_{n}$ on $\Sigma_{n}$ satisfying the following properties: (1) the curvature $K\left(g_{n}\right)$ is bounded by $\left|K\left(g_{n}\right)\right| \leq 1 ;(2)$ the injectivity radius of $\Sigma_{n}$ with respect to $g_{n}$ is not less than 1 ; $(3) g_{n}$ converges uniformly to a complete metric $g_{\infty}$ on $\Sigma_{\infty} \backslash\{$ nodes $\}$ in $C^{6}$-topology; (4) the limit metric $g_{\infty}$ is quasi-isometric to the standard Euclidean metric on cylinder near each node of $\Sigma_{\infty}$, i.e. $C^{-1} g_{\text {cylinder }} \leq$ $g_{\infty} \leq C g_{\text {cylinder }}$ for some constant $C \geq 1$. The existence of the metric $g_{n}$ is well-known. Note that since the domain spaces are 2-dimensional, harmonicity is invariant under conformal changes of domain, and hence $u_{n}$ is harmonic with respect to the metric $g_{n}$. In particular, the Dirichlet energy of $u_{n}$ is unchanged under conformal transformations.

The following $\epsilon$-regularity theorem is due to Sacks-Uhlenbeck [SU1] and SchoenUhlenbeck $[\mathrm{ScU}]$. Note that we choose the geodesic ball of unit size for simplicity.

Lemma 2.1. There exist constants $\epsilon_{0}>0$ and $C>0$ depending only on $M$, the injectivity radius and the curvature bound for the metric $g$ such that if $u$ is harmonic on $B_{2}\left(x_{0}, g\right) \subset M$ and

$$
\int_{B_{1}(x)}|\nabla u|_{g}^{2} d v_{g} \leq \epsilon_{0}
$$

then

$$
\sup _{B_{\frac{1}{2}}\left(x_{0}, g\right)}|\nabla f|_{g}^{2} \leq C \int_{B_{1}\left(x_{0}, g\right)}|\nabla u|_{g}^{2} d v_{g}
$$

We will also need the Removable Singularity Theorem of Sacks-Uhlenbeck [SU1]:

Lemma 2.2. Let $f$ be any continuous harmonic map from a punctured disk $D \backslash\{0\}$ in a Riemann surface into a manifold $X$ with finite total energy. Then $f$ extends to a smooth harmonic map from $D$ to $X$.

Note that the regularity of $f$ improves from $C^{0}$ to $C^{\infty}$ is well known.

Set $r_{m}=2^{-m}$ where $m \in \mathbb{Z}^{+}$. Define

$$
E_{m, n}=\left\{\left.x \in \Sigma\left|\int_{B_{r_{m}}\left(x, g_{n}\right)}\right| \nabla u_{n}\right|_{g_{n}} ^{2} d v_{g_{n}} \geq \epsilon_{0}\right\}
$$


where $\epsilon_{0}$ is given in Lemma 1. It is clear that $E_{m_{2}, n} \subseteq E_{m_{1}, n}$ if $m_{2} \geq m_{1}$. The following claim is proved in p. 281 in [RT]:

Claim. For $n$ sufficiently large, each $E_{m, n}$ can be covered by a collection of balls $B_{8 r_{m}}\left(x_{n 1}, g_{n}\right), \ldots, B_{8 r_{m}}\left(x_{n s}, g_{n}\right)$ where $s$ is independent of $n$.

We may assume that the sequence $\left\{x_{n k}\right\}$ converges to a point $x_{k}$ for $k=1, \ldots, s$ without loss of generality. Also, we may assume that the injectivity radius in $g_{n}$ is larger than 1 . Within each ball $B_{\frac{1}{8}}\left(x_{n i}, g_{n}\right)$ for $i=1, \ldots, s$, there is a point $y_{n i}$ so that

$$
e_{n i}=\left|\nabla u_{n}\right|_{g_{n}}^{2}\left(y_{n i}\right)=\max _{y \in B_{1}\left(x_{n i}, g_{n}\right)}\left|\nabla u_{n}\right|_{g_{n}}^{2}(y) .
$$

Then we may assume that

$$
\lim _{n \rightarrow \infty} e_{n i}=\infty .
$$

Define a new metric on $B_{1}\left(x_{n i}, g_{n}\right)$ by

$$
g^{\prime}{ }_{n i}=\frac{e_{n i} g_{n}}{\phi_{n i}\left(1+16 e_{n i} r^{2}\right)}
$$

where $r$ is the distance function from $y_{n i}$ of $g_{n}$ and $\phi$ is a cut-off function defined by

$$
\phi_{n i}(t)= \begin{cases}t & \text { if } t \leq 1+e_{n i} \\ e_{n i} & \text { if } t \geq 2+e_{n i}\end{cases}
$$

and

$$
\left|\phi_{n i}^{\prime}(t)\right| \leq 1,\left|\phi_{n i}^{\prime \prime}(t)\right| \leq C \text { for all } t
$$

for some constant $C$ independent of $n$. We change the metric $g_{n}$ on all balls $B_{1}\left(x_{n i}, g_{n}\right)$ for $i=1, \ldots, s$ to get a new metric $g_{n}^{\prime}$ on $\Sigma$. With respect to the new metric $g_{n}^{\prime}$, we have

$$
\begin{aligned}
& \left|\nabla u_{n}\right|_{g_{n}^{\prime}}^{2}\left(y_{n i}\right)=1, \\
& \left|\nabla u_{n}\right|_{g_{n}^{\prime}}^{2}(y) \leq \phi_{n i}\left(1+16 e_{n i} r^{2}\right) .
\end{aligned}
$$

Notice that $g_{n}^{\prime}$ coincides with $g_{n}$ in the annulus $B_{1}\left(x_{n i}, g_{n}\right) \backslash B_{\frac{1}{2}}\left(x_{n i}, g_{n}\right)$ and $\left|\nabla u_{n}\right|_{g_{n}}^{2}$ is uniformly bounded over this annulus. Recall if $h^{\prime}=e^{f} h$ is a conformal change of metrics in 2-dimensional manifold, then the Ricci tensors are related by

$$
\operatorname{Ric}\left(h^{\prime}\right)=\operatorname{Ric}(h)+\left(\frac{1}{2} \triangle_{h} f\right) h
$$

Therefore it is easy to see that the curvature $K\left(g_{n}^{\prime}\right)$ is also bounded from above and below on $B_{1}\left(x_{n i}, g_{n}^{\prime}\right)$ by noting that $f=\ln e_{n i}-\ln \phi_{n i}$ for our choice of conformal change. Since $u_{n}$ is an energy minimizer, the standard elliptic estimates imply

$$
\int_{B_{1}\left(x_{n i}, g_{n}^{\prime}\right)}\left|\nabla u_{n}\right|_{g_{n}^{\prime}}^{2} d A_{g_{n}^{\prime}} \geq \delta
$$


where $\delta$ is a positive number independent of $n$. Next, if $\left|\nabla u_{n}\right|_{g_{n}^{\prime}}^{2}$ is not uniformly bounded on $B_{1}\left(x_{n i}, g_{n}\right)$, then there is a point $z_{n i} \in B_{\frac{1}{2}}\left(x_{n i}, g_{n}\right) \backslash B_{4}\left(y_{n i}, g_{n}^{\prime}\right)$, such that

$$
\left|\nabla u_{n}\right|_{g_{n}}^{2}\left(z_{n i}\right)=\sup _{B_{1}\left(x_{n i}, g_{n}\right)}\left|\nabla u_{n}\right|_{g_{n}^{\prime}}^{2}=e_{n i}^{\prime}
$$

and

$$
\lim _{n \rightarrow \infty} e_{n i}^{\prime}=\infty .
$$

Then we change the metric $g_{n}^{\prime}$ conformally in a small neighborhood of $z_{n i}$ as we just did for $g_{n}$ to obtain a new metric $g_{n}^{\prime \prime}$. We have $B_{1}\left(y_{n i}, g_{n}^{\prime}\right) \cap B_{1}\left(z_{n i}, g_{n}^{\prime \prime}\right)=\emptyset$ and

$$
\int_{B_{1}\left(z_{n i}, g_{n}^{\prime \prime}\right)}\left|\nabla u_{n}\right|_{g_{n}^{\prime \prime}}^{2} d A_{g_{n}^{\prime \prime}} \geq \delta
$$

Again, if $\left|\nabla u_{n}\right|_{g_{n}^{\prime \prime}}^{2}$ is not uniformly bounded, we repeat the above arguments and obtain $w_{n i}$ and so on. Note that each time we use a positive amount of energy, which is at least $\delta$. But the total energy is fixed under conformal transformations on the domain surface. Therefore this process will stop after finitely many steps. In this way, we find a metric and with respect to it the energy density of $u_{n}$ is uniformly bounded. By Courant-Lebesque lemma (cf. [J1]), a subsequence of $\left\{u_{n}\right\}$ will converge to a harmonic map as $n \rightarrow \infty$ from $\Sigma_{\infty}$ union with some 2-spheres each with a point (the blow up point of $\left|\nabla u_{n}\right|_{g_{n}}^{2}$ ) deleted. Then the Removable Singularity Theorem implies the limit harmonic map extends to the whole 2-spheres.

By the $\epsilon$-regularity theorem for harmonic maps and Arzela-Ascoli theorem, $u_{n}$, taking a subsequence if necessary, converges strongly to a harmonic map $u_{\infty}$ on $\Sigma \backslash\left\{x \in \Sigma \mid d_{g_{n}}\left(x, E_{m, \infty}\right)<4 r_{m}\right\}$ where

$$
E_{m, \infty}=\bigcap_{n}^{\infty} E_{m, n} \subseteq \lim _{n \rightarrow \infty} \bigcup_{i=1}^{s} B_{8 r_{m}}\left(x_{i}, g_{n}\right) .
$$

We set

$$
\tau=12 r_{m}
$$

On the other hand, for any given constant $K$,

$$
g_{n}^{\prime} \geq \frac{e_{n}}{1+16 K^{2}} g_{n}
$$

if the distance $r$ from $y_{n i}$ in $g_{n}$ satisfying

$$
r \leq e_{n i}^{-\frac{1}{2}} K
$$

Now we decompose $\left(\Sigma, g_{n}\right)$ into three parts: the regular part

$$
\Sigma \backslash\left\{x \in \Sigma \mid d_{g_{n}}\left(x, E_{m, n}\right)<4 r_{m}\right\},
$$


the bubbling part

$$
\bigcup_{i=1}^{s} B_{e_{n i}^{-\frac{1}{2}} K}\left(y_{n i}, g_{n}\right)
$$

and the connecting part

$$
A_{n i}(\tau, K)=\left\{x \in B_{1}\left(x_{n i}, g_{n}\right): e_{n i}^{-\frac{1}{2}} K \leq\left|x-y_{n i}\right|_{g_{n}} \leq \tau\right\} .
$$

Our main interests will be focused on the connecting part. Along a connecting annulus, we consider the map

$$
f:(t, \theta) \rightarrow(-\log t, \theta)
$$

which maps $A_{n i}$ conformally with factor $t^{-2}$ to a cylinder

$$
\mathcal{C}_{n i}(\tau, K)=\left[-\log \tau,-\log \left(e_{n i}^{-\frac{1}{2}} K\right)\right] \times \mathbb{S}^{1}
$$

with coordinate $(t, \theta)$ and metric

$$
d s^{2}=d t^{2}+d \theta^{2} .
$$

A harmonic map $u$ from the flat cylinder $\mathcal{C}=[0, R] \times \mathbb{S}^{1}$ into $M$, which is isometrically embedded in some Euclidean space $\mathbb{R}^{N}$ takes the form of

$$
\frac{\partial^{2} u}{\partial t^{2}}+\frac{\partial^{2} u}{\partial \theta^{2}}+B_{i j}(u)\left(\frac{\partial u^{i}}{\partial t} \frac{\partial u^{j}}{\partial t}+\frac{\partial u^{i}}{\partial \theta} \frac{\partial u^{j}}{\partial \theta}\right)=0
$$

where $B$ is the second fundamental form of $M$ in $\mathbb{R}^{N}$. In particular, if a harmonic map from $[0, R] \times \mathbb{S}^{1}$ into $M$ is $\theta$-independent, i.e., $\frac{\partial u}{\partial \theta}=0$, then it satisfies the equation for geodesics in $M$

$$
\frac{\partial^{2} u}{\partial t^{2}}+B_{i j}(u) \frac{\partial u^{i}}{\partial t} \frac{\partial u^{j}}{\partial t}=0 .
$$

If the length of $\mathcal{C}$ is $R$, then we divide $\mathcal{C}$ into $m$ equal length pieces and set

$$
I_{i}=\{(t, \theta):(i-1) l \leq t \leq i l\}
$$

where $R=m l$ and $i=1, \ldots, m$. If $w_{1}$ and $w_{2}$ are two solutions of (2.1), we put

$$
w=w_{1}-w_{2}
$$

here we embed $M$ into some Euclidean space isometrically $\mathbb{R}^{N}$ and set

$$
\|w\|_{i}^{2}=\int_{I_{i}}|w|^{2} d t d \theta
$$


It is easy to check that $w$ satisfies

$$
\frac{\partial^{2} w}{\partial t^{2}}+\frac{\partial^{2} w}{\partial \theta^{2}}+A \cdot \nabla w+B \cdot w=0
$$

where

$$
|A| \leq C\left(\left|\nabla w_{1}\right|+\left|\nabla w_{2}\right|\right) \text { and }|B| \leq C \min \left\{\left|\nabla w_{1}\right|^{2},\left|\nabla w_{2}\right|^{2}\right\}
$$

for some uniform constant $C$. We now prove a result regarding the asymptotic behavior of solutions of equation (2.5).

Proposition 2.3. Suppose that $m$ and $l$ are fixed and $l$ is large $($ say $l>\ln 2)$. Let $v$ be a solution of (2.5). There is a constant $\delta_{0}$ such that if and $|A|_{L^{2}(\mathcal{C})}+|B|_{L^{2}(\mathcal{C})} \leq$ $\delta_{0}$, then we have the following alternatives:

(i) if $\|v\|_{i} \geq e^{\frac{l}{2}}\|v\|_{i+1}$, then $\|v\|_{i-1} \geq e^{\frac{l}{2}}\|v\|_{i}$;

(ii) if $\|v\|_{i} \geq e^{\frac{l}{2}}\|v\|_{i-1}$, then $\|v\|_{i+1} \geq e^{\frac{l}{2}}\|v\|_{i}$;

(iii) if

$$
\int_{I_{i}} v d t d \theta=0
$$

and

$$
\int_{I_{i}} t v d t d \theta=0
$$

then either $\|v\|_{i} \leq e^{-\frac{l}{2}}\|v\|_{i+1}$ or $\|v\|_{i} \leq e^{-\frac{l}{2}}\|v\|_{i-1}$.

Proof. First, we consider the limit case when $|A|_{L^{2}(\mathcal{C})}+|B|_{L^{2}(\mathcal{C})} \leq \delta \rightarrow 0$. For each $\delta>0$, we denote the solution of $(2.5)$ with $|A|_{L^{2}(\mathcal{C})}+|B|_{L^{2}(\mathcal{C})} \leq \delta$ by $v_{\delta}$. Suppose that (i) does not hold. So we have

$$
\begin{aligned}
& \left\|v_{\delta}\right\|_{i} \geq e^{\frac{l}{2}}\left\|v_{\delta}\right\|_{i+1} \\
& \left\|v_{\delta}\right\|_{i} \geq e^{-\frac{l}{2}}\left\|v_{\delta}\right\|_{i-1} .
\end{aligned}
$$

We normalize $v_{\delta}$ such that $\left\|v_{\delta}\right\|_{i}=1$. Then by taking a subsequence of $v_{\delta}$ in $\delta \rightarrow 0$ and by the standard elliptic estimates, we may assume that $v_{\delta}$ converge to a harmonic map $v_{0}$ from the cylinder into Euclidean space $\mathbb{R}^{N}$

$$
\frac{\partial^{2} v_{0}}{\partial t^{2}}+\frac{\partial^{2} v_{0}}{\partial \theta^{2}}=0
$$

and

$$
\begin{aligned}
& \left\|v_{0}\right\|_{i} \geq e^{\frac{l}{2}}\left\|v_{0}\right\|_{i+1} \\
& \left\|v_{0}\right\|_{i} \geq e^{-\frac{l}{2}}\left\|v_{0}\right\|_{i-1} .
\end{aligned}
$$


Therefore we only need to prove the proposition for a solution $v_{0}$ of (2.8). By separation of variables, the expansion of $v_{0}$ is

$$
v_{0}=a_{0}+b_{0} t+\sum_{k \neq 0}\left(a_{k} \cos k \theta+b_{k} \sin k \theta\right) e^{k t} .
$$

It follows that

$$
\begin{aligned}
& \left\|v_{0}\right\|_{i}^{2}=\int_{(i-1) l}^{i l} \int_{0}^{2 \pi}\left(a_{0}+b_{0} t\right)^{2} d t d \theta \\
& +\sum_{k=1}^{\infty} \int_{(i-1) l}^{i l}\left(e^{2 k t}\left(a_{k}^{2}+a_{-k}^{2}\right)+e^{-2 k t}\left(b_{k}^{2}+b_{-k}^{2}\right)\right) d t \int_{0}^{2 \pi} \cos ^{2} k \theta d \theta \\
& =2 \pi\left(a_{0}^{2} l+a_{0} b_{0} l^{2}(2 i-1)+\frac{1}{3} b_{0}^{2} l^{3}\left(3 i^{2}-3 i+1\right)\right) \\
& +\frac{\pi}{2} \sum_{k=1}^{\infty}\left(k^{-1} e^{2(i-1) k l}\left(e^{2 k l}-1\right)\left(a_{k}^{2}+a_{-k}^{2}\right)\right. \\
& \left.+k^{-1} e^{-2(i-1) k l}\left(1-e^{-2 k l}\right)\left(b_{k}^{2}+b_{-k}^{2}\right)\right) .
\end{aligned}
$$

If (i) does not hold, then

$$
\left\|v_{0}\right\|_{i}^{2} \geq \frac{1}{2}\left(e^{l}\left\|v_{0}\right\|_{i+1}^{2}+e^{-l}\left\|v_{0}\right\|_{i-1}^{2}\right)
$$

Then applying the recursive formula (2.10) for $i-1, i, i+1$ to the above inequality, we obtain

$$
\begin{aligned}
& 2 \sum_{k=1}^{\infty}\left(k^{-1} e^{2(i-1) k l}\left(e^{2 k l}-1\right)\left(a_{k}^{2}+a_{-k}^{2}\right)+k^{-1} e^{-2(i-1) k l}\left(1-e^{-2 k l}\right)\left(b_{k}^{2}+b_{-k}^{2}\right)\right) \\
\geq & \sum_{k=1}^{\infty}\left(k^{-1}\left(e^{l+2 k l}+e^{-l-2 k l}\right) e^{2(i-1) k l}\left(e^{2 k l}-1\right)\left(a_{k}^{2}+a_{-k}^{2}\right)\right) \\
& +\sum_{k=1}^{\infty}\left(k^{-1}\left(e^{l-2 k l}+e^{-l+2 k l}\right) e^{-2(i-1) k l}\left(1-e^{-2 k l}\right)\left(b_{k}^{2}+b_{-k}^{2}\right)\right) .
\end{aligned}
$$

It is impossible as $e^{l+2 k l}+e^{-l-2 k l}>2$ and $e^{l-2 k l}+e^{-l+2 k l}>2$. Similarly, one can prove (ii). For (iii), we have $a_{0}=0, b_{0}=0$. Now

$$
\begin{aligned}
\left\|v_{0}\right\|_{i}^{2} & =\frac{\pi}{2} \sum_{k=1}^{\infty}\left(k^{-1} e^{2(i-1) l k}\left(e^{2 k l}-1\right)\left(a_{k}^{2}+a_{-k}^{2}\right)\right. \\
& \left.+k^{-1} e^{-2(i-1) k l}\left(1-e^{-2 k l}\right)\left(b_{k}^{2}+b_{-k}^{2}\right)\right)
\end{aligned}
$$


and

$$
\begin{aligned}
& \frac{1}{2} e^{-l}\left(\left\|v_{0}\right\|_{i+1}^{2}+\left\|v_{0}\right\|_{i-1}^{2}\right) \\
= & \frac{\pi}{4} e^{-l} \sum_{k=1}^{\infty}\left(k^{-1} e^{2(i-1) k l+2 k l}\left(e^{2 k l}-1\right)\left(a_{k}^{2}+a_{-k}^{2}\right)\right. \\
& \left.+k^{-1} e^{-2(i-1) k l+2 k l}\left(1-e^{2 l}\right)\left(b_{k}^{2}+b_{-k}^{2}\right)\right) .
\end{aligned}
$$

By term by term comparison, it is easy to see that if $l>\ln 2$

$$
\left\|v_{0}\right\|_{i}^{2} \leq \frac{1}{2} e^{-l}\left(\left\|v_{0}\right\|_{i+1}^{2}+\left\|v_{0}\right\|_{i-1}^{2}\right) .
$$

Therefore we have shown the proposition for harmonic functions. Now the general case follows since $v_{\delta}$ converges to $v_{0}$ in $W^{2,2}$ (see also the proof of Corollary 2.4 below).

In fact, the same method yields the following more general result (cf. [QT]).

Corollary 2.4. Let $v$ satisfy

$$
\triangle v+A^{i j} D_{i j}^{2} v+B \cdot \nabla v+C \cdot v=f
$$

on the cylinder $\mathcal{C}=[0, m l] \times S^{1}$. Then for a given $p>1$, there exists a positive number $\delta_{1}$ such that if

$$
\|f\|_{L^{p}(\mathcal{C})} \leq \delta_{1}\left(\max _{1 \leq i \leq m}\|v\|_{W^{1,2}\left([(i-1) l, i l] \times S^{1}\right)}^{2}+\sup _{\mathcal{C}}|v|^{2}\right)
$$

and

$$
\|A\|_{C^{0}(\mathcal{C})}+\|B\|_{L^{\infty}(\mathcal{C})}+\|C\|_{L^{\infty}(\mathcal{C})} \leq \delta_{1},
$$

then for $2 \leq i \leq m-1$ the following alternatives hold:

(i) if $\|v\|_{i} \geq e^{\frac{l}{2}}\|v\|_{i+1}$, then $\|v\|_{i-1} \geq e^{\frac{l}{2}}\|v\|_{i}$;

(ii) if $\|v\|_{i} \geq e^{\frac{l}{2}}\|v\|_{i-1}$, then $\|v\|_{i+1} \geq e^{\frac{l}{2}}\|v\|_{i}$;

(iii) if

$$
\int_{I_{i}} v d t d \theta \leq \delta_{1}\left(\|v\|_{W^{1,2}(\mathcal{C})}^{2}+\sup _{\mathcal{C}}|v|^{2}\right)
$$

and

$$
\int_{I_{i}} t v d t d \theta \leq \delta_{1}\left(\|v\|_{W^{1,2}(\mathcal{C})}^{2}+\sup _{\mathcal{C}}|v|^{2}\right),
$$

then either $\|v\|_{i} \leq e^{-\frac{l}{2}}\|v\|_{i+1}$ or $\|v\|_{i} \leq e^{-\frac{l}{2}}\|v\|_{i-1}$. 
Proof. If the corollary were not true, then there would be a sequence of $\delta_{k} \rightarrow 0$ and a sequence of solutions $v_{k}$ of the equation (2.11) with

$$
\|f\|_{L^{p}} \leq \delta_{k}\left(\max _{1 \leq i \leq m}\left\|v_{k}\right\|_{W^{1,2}\left([(i-1) l, i l] \times S^{1}\right)}^{2}+\sup _{\mathcal{C}}\left|v_{k}\right|^{2}\right)
$$

and

$$
\|A\|_{C^{0}(\mathcal{C})}+\|B\|_{L^{\infty}(\mathcal{C})}+\|C\|_{L^{\infty}(\mathcal{C})} \leq \delta_{k} .
$$

Let us assume (i) is not true for $v_{k}$ (the other case can be argued similarly). Therefore for some $i, 2 \leq i \leq m-2$, we have

$$
\begin{aligned}
& \text { (a) }\left\|v_{k}\right\|_{i} \geq e^{l / 2}\left\|v_{k}\right\|_{i+1}, \\
& \text { (b) }\left\|v_{k}\right\|_{i}>e^{-l / 2}\left\|v_{k}\right\|_{i-1} .
\end{aligned}
$$

Normalize $v_{k}$ over $[(i-1) l,(i+2) l] \times S^{1}$ by dividing the norm of $v_{k}$ over the middle interval $I_{i+1}$

$$
v_{k}^{\prime}=\frac{v_{k}}{\left\|v_{k}\right\|_{W^{1,2}\left(I_{i+1}\right)}+\sup _{I_{i+1}}\left|v_{k}\right|} .
$$

Now (a), (b) and the elliptic estimates assert that the norms of $v_{k}^{\prime}$ over $I_{i}, I_{i+2}$ are bounded above by that over $I_{i+1}$ times a uniform constant (depends on $e^{l}$ ). The interior estimates (cf. [GT]) then imply that on every domain $[(i-1) l+\epsilon,(i+2) l-$ $\epsilon] \times S^{1}$ for sufficiently small positive $\epsilon$ there exists a subsequence of $v_{k}^{\prime}$ converges to a harmonic function. So by a diagonal process, a subsequence of $v_{k}^{\prime}$ converges to a harmonic function $v_{0}^{\prime}$ on $((i-1) l,(i+2) l) \times S^{1}$ and $v_{0}^{\prime}$ satisfies (a) and (b) ("> " replaced by " $\geq "$ ). But the norm of $v_{0}^{\prime}$ on $I_{i+1}$ is 1 hence $v_{0}^{\prime}$ is nonzero. We then obtain a contradiction to Proposition 2.3.

Along the cylinder, there is a unique solution of (2.2),i.e., $\theta$-independent solution of (2.1), for any given initial data, and this solution is a geodesic in $M$. We will show that if $\delta$ in (2.3) is sufficiently small then any solution of (2.1) is very close to a geodesic. Recall that harmonicity and the Dirichlet integral is invariant under conformal transformations if the domain is 2-dimensional. For the harmonic map $u_{n}$ the composition $u_{n} \circ f^{-1}$ is harmonic on the corresponding cylinder $f\left(A_{n i}(\tau, K)\right)$. For the sake of simplicity, we will still use $u_{n}$ to denote the harmonic maps on the cylinders. After rescaling the metric

$$
\left\{\begin{array}{l}
\left\|\nabla u_{n}\right\|_{C^{0}\left(\Sigma, g_{n}^{\prime}\right)} \leq C \\
\left\|\nabla u_{n}\right\|_{C^{0}(\mathcal{C})} \leq \epsilon .
\end{array}\right.
$$

for some positive constant $\epsilon$ which is so small that Proposition 2.5 and Proposition 2.6 can be applied (refer to Proposition 2.5 and Proposition 2.6).

Proposition 2.5. There is a constant $\epsilon>0$ such that if $\left\|\nabla u_{n}\right\|_{C^{0}(\mathcal{C})}<\epsilon$, then on each $I_{i}$, there exists a curve in $M$ given by a solution $\tilde{u}_{n}$ of (2.2) such that $\tilde{u}_{n}$ 
and satisfies the following normalization

$$
\begin{aligned}
& \int_{I_{i}}\left(u_{n}-\tilde{u}_{n}\right) d t d \theta=0 \\
& \int_{I_{i}} t\left(u_{n}-\tilde{u}_{n}\right) d t d \theta=0
\end{aligned}
$$

and

$$
\left\|\nabla \tilde{u}_{n}\right\|_{C^{0}([i l,(i+1) l])} \leq C \epsilon
$$

where $C$ is a positive constant depending only on $M$.

Proof. A $\theta$-independent solution $v_{n}$ of (2.8) is of the form

$$
v_{n}(t)=p_{n}+t q_{n}
$$

where $p_{n}$ is a point in $M$ and $q_{n}$ is a fixed vector in $T_{p_{n}} M$. In fact, $p_{n}$ and $q_{n}$ can be viewed as vectors in $\mathbb{R}^{N}$ where $M$ is isometrically embedded. The two constrains on $\tilde{u}$ can be interpreted as initial values as follows. Integrating over $I_{i}$,

$$
\begin{aligned}
2 \pi\left(l p_{n}+\frac{1}{2} l^{2} q_{n}\right) & =\int_{S^{1}} \int_{0}^{l} u_{n}(t, \theta) d t d \theta \\
2 \pi\left(\frac{1}{2} l^{2} p_{n}+\frac{1}{3} l^{3} q_{n}\right) & =\int_{S^{1}} \int_{0}^{l} t u_{n}(t, \theta) d t d \theta .
\end{aligned}
$$

Since $l \neq 0$, there exists a unique solution $\left(p_{n}, q_{n}\right)$ for the system. The first derivative at $v_{n}$ of the functional $\partial_{t}^{2}$ on $C^{\infty}\left([i l,(i+1) l], \mathbb{R}^{N}\right)$ is

$$
\left.\frac{d}{d s}\right|_{s=0} \partial_{t}^{2}\left(v_{n}(t)+s f(t)\right)=\partial_{t}^{2} f
$$

for any $f \in C^{\infty}\left([i l,(i+1) l], \mathbb{R}^{N}\right)$. It is straightforward to check that the first derivative of the functional is both surjective and injective on the subspace of $C^{\infty}\left([i l,(i+1) l], \mathbb{R}^{N}\right)$ whose elements satisfy (2.16) and (2.17). Then the Inverse Function Theorem implies that there is some constant $\delta_{2}>0$ such that if $|h|<\delta_{2}$ for $h \in C^{\infty}\left([i l,(i+1) l], \mathbb{R}^{N}\right)$ then the equation

$$
\partial_{t}^{2} f=h
$$

has a unique solution in $C^{\infty}\left([i l,(i+1) l], \mathbb{R}^{N}\right)$ with $(2.16),(2.17)$ satisfied. Next, since $|\nabla u| \leq \epsilon$ on $\mathcal{C}$ by assumption, the image of $I_{i}$ under $u$ is contained in a small convex region. In fact, there is a point $x_{0}$ in $M$ such that for any $(t, \theta) \in I_{i}$,

$$
\left|u_{n}(t, \theta)-x_{0}\right| \leq 2 l\left\|\nabla u_{n}\right\|_{C^{0}} \leq 2 l \epsilon
$$


If $\tilde{u}_{n}$ is a geodesic from $[i l,(i+1) l]$ to the same small region in $M$, it has constant speed, we have

$$
\left|\frac{d \tilde{u}_{n}}{d t}\right| \leq C \frac{2 l \epsilon}{l} \leq 2 C \epsilon
$$

Therefore if we pick $\epsilon$ sufficiently small in advance, we have

$$
\left|B_{i j}\left(\tilde{u}_{n}\right) \partial_{t} \tilde{u}_{n}^{i} \partial_{t} \tilde{u}_{n}^{j}\right| \leq C \epsilon<\delta_{2}
$$

where $C$ depends only on the second fundamental form of $M$ in $\mathbb{R}^{N}$, and the Inverse Function Theorem then asserts the existence of a geodesic which satisfying the two normalization conditions (2.16) and (2.17). This completes the proof of Proposition 2.5.

Proposition 2.6. Let $u_{n}, \tilde{u}_{n}$ be as before and $\left\|\nabla u_{n}\right\|_{C^{0}(\mathcal{C})}<\epsilon<C_{1} \delta_{1}$ where $\epsilon, \delta_{1}$ are given in Proposition 2.5 and Corollary 2.4 respectively and $C_{1}<1$ is some positive constant independent of $n$. Then there exists $i_{0} \geq 2$ such that

$$
\begin{gathered}
\left|u_{n}(t, \theta)-\tilde{u}_{n}(t)\right|+\left|\nabla u_{n}(t, \theta)-\nabla \tilde{u}_{n}(t)\right| \leq \delta_{1} C e^{-\frac{1}{2}(R-t),}, \text { if } t \geq i_{0} l, \\
\left|u_{n}(t, \theta)-\tilde{u}_{n}(t)\right|+\left|\nabla u_{n}(t, \theta)-\nabla \tilde{u}_{n}(t)\right| \leq \delta_{1} C e^{-\frac{1}{2} t}, \quad \text { if } t<i_{0} l
\end{gathered}
$$

where $C$ is a universal constant.

Proof. By Proposition 2.5 and (iii) in Corollary 2.4, for any fixed $i_{0} \geq 2$ either

$$
\left\|u_{n}-\tilde{u}_{n}\right\|_{i_{0}} \leq e^{-\frac{1}{2} l}\left\|u_{n}-\tilde{u}_{n}\right\|_{i_{0}+1}
$$

or

$$
\left\|u_{n}-\tilde{u}_{n}\right\|_{i_{0}} \leq e^{-\frac{1}{2} l}\left\|u_{n}-\tilde{u}_{n}\right\|_{i_{0}-1} .
$$

Suppose the first case is true. Then by (ii) in Proposition 2.4,

$$
\left\|u_{n}-\tilde{u}_{n}\right\|_{i+1} \geq e^{\frac{1}{2} l}\left\|u_{n}-\tilde{u}_{n}\right\|_{i}
$$

for any $i \geq i_{0}$. We claim

$$
\left\|u_{n}-\tilde{u}_{n}\right\|_{m} \leq \delta_{1}=\frac{\delta}{100} .
$$

If this were not the case, then there would be some $i>i_{0}$ such that

$$
\left\|u_{n}-\tilde{u}_{n}\right\|_{i} \geq \delta_{1} .
$$

On the other hand,

$$
\begin{aligned}
\left\|u_{n}-\tilde{u}_{n}\right\|_{i+1}^{2} & \leq\left\|u_{n}-\tilde{u}_{n}\right\|_{i}^{2}+\left\|\nabla\left(u_{n}-\tilde{u}_{n}\right)\right\|_{C^{0}}^{2} \cdot 2 \pi l \\
& \leq\left\|u_{n}-\tilde{u}_{n}\right\|_{i}^{2}+2 \pi l\left(1+C^{2}\right) \epsilon^{2} .
\end{aligned}
$$


It follows

$$
\begin{aligned}
\left(e^{l}-1\right) \delta_{1}^{2} & \leq\left(e^{l}-1\right)\left\|u_{n}-\tilde{u}_{n}\right\|_{i}^{2} \\
& \leq\left\|u_{n}-\tilde{u}_{n}\right\|_{i+1}^{2}-\left\|u_{n}-\tilde{u}_{n}\right\|_{i}^{2} \\
& \leq 2 \pi l\left(1+C^{2}\right) \epsilon^{2} .
\end{aligned}
$$

This is impossible since $\epsilon<C_{1} \delta_{1}$ and we can require in advance that

$$
C_{1}^{2}>\frac{2 \pi l\left(1+C^{2}\right)}{e^{l}-1}
$$

Therefore by iteration, we have

$$
\begin{aligned}
\left\|u_{n}-\tilde{u}_{n}\right\|_{i_{0}} & \leq e^{-\frac{1}{2}\left(m-i_{0}\right) l}\left\|u_{n}-\tilde{u}_{n}\right\|_{m} \\
& \leq \delta_{1} e^{-\frac{1}{2}\left(m-i_{0}\right) l} .
\end{aligned}
$$

For the second case, we can argue similarly. Indeed, if

$$
\left\|u_{n}-\tilde{u}_{n}\right\|_{i_{0}} \leq e^{-\frac{1}{2} l}\left\|u_{n}-\tilde{u}_{n}\right\|_{i_{0}-1}
$$

we conclude first that

$$
\left\|u_{n}-\tilde{u}_{n}\right\|_{0} \leq \delta_{1}
$$

and then

$$
\left\|u_{n}-\tilde{u}_{n}\right\|_{i_{0}} \leq \delta_{1} e^{-\frac{1}{2} i_{0} l}
$$

Choose $i_{0}$ to be the smallest one so that

$$
\left\|u_{n}-\tilde{u}_{n}\right\|_{i_{0}} \leq e^{-\frac{1}{2} l}\left\|u_{n}-\tilde{u}_{n}\right\|_{i_{0}+1} .
$$

Note that after this $i_{0}$, the above inequality remains to hold. Then the foregoing discussions show the following. As $m \rightarrow \infty$ or equivalently $R=m l$ goes to infinity, $u$ decays exponentially over $I_{i_{0}}$ to $\tilde{u}_{n}$ from left if $i \leq i_{0}$ and from right if $i \geq i_{0}$.

The difference of two harmonic maps, especially $u_{n}-\tilde{u}_{n}$, satisfies differential equation (2.5) where the coefficients $A$ and $B$ may depend on $w$ but

$$
\|A\|_{C^{0}},\|B\|_{C^{0}} \leq C \epsilon
$$

for some constant $C$ depending only on $M$. Then by the $L^{p}$-theory in the elliptic partial differential equations ([GT]) for $p=2,\left\|u_{n}-\tilde{u}_{n}\right\|_{W^{2,2}}$ can be bounded by the $L^{2}$-norm of $u_{n}-\tilde{u}_{n}$. The Sobolev embedding theorem implies that the $\left\|u_{n}-\tilde{u}_{n}\right\|_{C^{0}}$ is bounded by the $L^{2}$-norm. So for any $p>2,\left\|u_{n}-\tilde{u}_{n}\right\|_{L^{p}}$ is bounded by $\left\|u_{n}-\tilde{u}_{n}\right\|_{L^{2}}$. Apply the $L^{p}$-theory again, we see that $\left\|u_{n}-\tilde{u}_{n}\right\|_{W^{2, p}}$ is bounded by $\left\|u_{n}-\tilde{u}_{n}\right\|_{L^{p}}$, hence by $\left\|u_{n}-\tilde{u}_{n}\right\|_{L^{2}}$. Applying the Sobolev embedding theorem 
again, so the $C^{1}$-norm of $u-\tilde{u}$ is bounded by $\left\|u_{n}-\tilde{u}_{n}\right\|_{L^{2}}$. Then by Proposition 2.4 and Proposition 2.5, we conclude

$$
\begin{aligned}
& \left|u_{n}(t, \theta)-\tilde{u}_{n}(t)\right|+\left|\nabla u_{n}(t, \theta)-\nabla \tilde{u}_{n}(t)\right| \leq C \delta_{1} e^{-\frac{1}{2}(R-t)}, \quad \text { if } t \geq i_{0} l \\
& \left|u_{n}(t, \theta)-\tilde{u}_{n}(t)\right|+\left|\nabla u_{n}(t, \theta)-\nabla \tilde{u}_{n}(t)\right| \leq C \delta_{1} e^{-\frac{1}{2} t}, \quad \text { if } t<i_{0} l .
\end{aligned}
$$

Now Proposition 2.6 is proved.

The rescaling procedure results in that $R$ tends to infinity as $n$ does. Within any fixed distance, measured in $t$, from each of the two ends of the cylinders, the standard elliptic estimates imply that $u_{n}$, a subsequence if necessary, converges to a limit. On the middle portion of the cylinders, we have just proved that $\|u-\tilde{u}\|_{C^{1}}$ decays exponentially in $t$. Therefore we have shown that there is a harmonic map $u^{j}$ from $\Sigma_{j} \cup \mathbb{S}^{2}$ 's into $M$ and $u^{j}$ converges to geodesics in $M$ along the connecting cylinders.

Next, we need to show that these geodesics all have finite length. Note that finiteness of the length of the geodesics does not follow simply from smallness of energy on the necks. It requires arguments involving global properties of the ambient manifold. However, if $M$ is simply connected there is a direct proof of the finiteness by constructing distance contracting maps on $M$ (cf. [J1]).

In fact, since $M$ is simply connected, there exists a map $f: M \rightarrow B_{r}(0) \subset M$, such that

$$
\begin{cases}d(f(x), f(y)) \leq d(x, y), & \text { for all } x, y \in M, \\ f(x)=x, & \text { if } x \in B_{r}(0) .\end{cases}
$$

Now if the length of a connecting geodesic $\gamma$ is very large, then there are two points $p, q \in \gamma$ satisfying (1) the length of the portion of $\gamma$ from $p$ to $q$ is large; (2) the distance between $p$ and $q$ is small in $M$, say $p, q \in B_{r}(0)$ (otherwise we move the center of the ball). Take a connecting cylinder $\mathcal{C}$ such that $u_{n}(\mathcal{C})$ converges to $\gamma$. So for sufficiently large $n$, there are points $p_{n}, q_{n} \in u_{n}(\mathcal{C}) \cap B_{r}(0)$, and the portion of $u_{n}(\mathcal{C})$ from $p_{n}$ to $q_{n}$ is very long. Without loss of generality, we may assume that

$$
r<\frac{1}{2} \min \left\{\frac{\pi}{2 K}, \text { injectivity radius of } 0\right\}
$$

where $K$ is the upper bound of the length of the curvature on $B_{2 r}(0)$. It is clear

$$
\left|\nabla\left(f \circ u_{n}\right)\right| \leq\left|\nabla u_{n}\right|
$$

since $f$ is distance contracting. Therefore

$$
E\left(f \circ u_{n}\right) \leq E\left(u_{n}\right) .
$$

In particular, strict inequality holds if $u_{n}(x)$ does not belong to $B_{r}(0)$ for some $x \in \mathcal{C}$. Recall that $u_{n}: \mathcal{C} \rightarrow M$ is energy minimizing among all maps from $\mathcal{C}$ 
to $M$ which send $\partial \mathcal{C}$ into $B_{r}(0)$ and homotopic to $u_{n}$. Obviously, $f \circ u_{n}$ belongs to this class of mappings. It then follows that $u_{n}(\mathcal{C}) \subset B_{r}(0)$. Therefore the geodesic $\gamma$ from $p$ to $q$ lies inside $B_{r}(0)$. So the length of $\gamma$ cannot be large since otherwise we can reduce the energy of $u_{n}$. We point out that the above arguments for the simply connected case holds for maps which minimize the perturbed energy functional also. Now let us consider the general case.

Proposition 2.7. The geodesics arising from the blowing up process all have finite length.

Proof. Recall that we have a sequence of energy minimizer $u_{n}$ converges to a minimizing harmonic map $u_{\infty}$ from $\Sigma_{\infty} \cup_{j=1}^{m} \mathbb{S}_{j}^{2}$ to $M$. We will derive estimates on the Dirichlet energy and length on a long connecting cylinder $\mathcal{C}$ first. As $n \rightarrow \infty, u_{n}(\mathcal{C})$ converges to a geodesic $\gamma$ pointwise exponentially. In the sequel, we will also use $C$ to denote positive uniform constants, and this should not cause any confusions with the notion for cylinders. By Proposition 2.6,

$$
\begin{gathered}
\left|\frac{\partial u_{n}}{\partial \theta}(t, \theta)\right| \leq C \delta_{1} e^{-\frac{1}{2}(R-t)}, \text { if } t \geq i_{0} l, \\
\left|\frac{\partial u_{n}}{\partial \theta}(t, \theta)\right| \leq C \delta_{1} e^{-\frac{1}{2} t}, \quad \text { if } t<i_{0} l .
\end{gathered}
$$

Suppose that the speed of the geodesic $\gamma$ is $\epsilon_{n}$ when it is parameterized by $t \in$ $\left[0, R_{n}\right]$. We are interested in the case when there is a lower bound on the length of $\gamma$, namely for some $C>0$,

$$
\epsilon_{n} R_{n}>C .
$$

Notice that in Proposition 2.6, the first estimate holds for large $t$, so if we let $t$ decrease to $t_{0}=i_{0} l$ from right, we still have the exponential decay estimate as in the second estimate. In fact, if $i_{0} l \leq \frac{R_{n}}{2}$, we use the first inequality in Proposition 2.6 for $t \in\left[\frac{R_{n}}{2}, \frac{3 R_{n}}{4}\right]$ and if $i_{0} l \geq \frac{R_{n}}{2}$ we use the second inequality in Proposition 2.6 for $t \in\left[\frac{R_{n}}{4}, \frac{R_{n}}{2}\right]$. So we have

$$
\left|\frac{\partial u_{n}}{\partial t}(t, \theta)-\frac{\partial \gamma}{\partial t}\right| \leq C \delta_{1} e^{-\frac{C}{\epsilon_{n}}}
$$

for $t$ belongs to the intervals described above. The Dirichlet energy of $u_{n}$ on $\mathcal{C}=\left[\frac{R_{n}}{2}, \frac{3 R_{n}}{4}\right] \times \mathbb{S}^{1}$ if $i_{0} l \leq \frac{R_{n}}{2}$ or on $\mathcal{C}_{2}=\left[\frac{R_{n}}{4}, \frac{R_{n}}{2}\right] \times \mathbb{S}^{1}$ if $i_{0} l \geq \frac{R_{n}}{2}$, then can be estimated by

$$
E\left(u_{n}, \mathcal{C}\right)=\frac{1}{4} \epsilon_{n}^{2} R_{n}+O\left(\delta_{1}^{2} e^{-\frac{2 C}{\epsilon_{n}}}+\delta_{1} \epsilon_{n} e^{-\frac{C}{\epsilon_{n}}}\right) R_{n} .
$$

The length of the cylinder $u_{n}(\mathcal{C})$, which is very close to the length of the geodesic $\gamma$ for $n$ large, is

$$
L\left(u_{n}(\mathcal{C})\right)=\frac{1}{4} \epsilon_{n} R_{n}+O\left(\delta_{1} e^{-\frac{C}{\epsilon_{n}}}\right) R_{n}
$$


Recall that we have the following situation: there are finitely many long cylinders connecting the regular parts and these cylinders converge to geodesics $\gamma_{1}, \ldots, \gamma_{k}$. These connecting cylinders arise either from the bubbling spheres or at the singularities of the stable curve compactification. We consider the following two cases.

Case 1. there is only one connecting geodesic $\gamma$, i.e., $k=1$. Assume the two end points of $\gamma$ are $x$ and $y$. Then we pick two points $p, q \in \gamma$ such that the length of the portion of $\gamma$ from $x$ to $p$ and from $y$ to $q$ is fixed. Denote the portions of $\gamma$ from $x$ to $p$ and from $y$ to $q$ by $\gamma(x, p)$ and $\gamma(y, q)$ respectively. Let $\gamma^{\prime}(p, q)$ be a distance minimizing geodesic in $M$ from $p$ to $q$. Now we have two curves from $x$ to $y$, namely $\gamma$ and $\gamma(x, p) \cup \gamma^{\prime}(p, q) \cup \gamma(y, q)$. We divide $\gamma(p, q)$ into unit length pieces $\gamma\left(s_{i}, s_{i+1}\right)$ where $i=0,1, \ldots$ and $s_{0}=p$. Since $M$ is compact, there exists a subsequence $\left\{s_{i_{\alpha}}\right\}$ of $\left\{s_{i}\right\}$ converges to a point $x_{0}$ in $M$. Since $M$ is path-connected, $\pi_{1}\left(M, \operatorname{rel}\left(s_{i}, s_{j}\right)\right)$, the group of homotopy equivalent maps from $[0,1]$ to $M$ with the same initial point $s_{i}$ and then same terminal point $s_{j}$, is isomorphic to $\pi_{1}(M, \operatorname{rel}(p, q))$ for $i \neq j$. Each curve $\gamma\left(s_{i_{\alpha}}, s_{i_{\alpha+1}}\right)$ represents a non-trivial element in $\pi_{1}\left(M, \operatorname{rel}\left(s_{i}, s_{j}\right)\right)$. This is because otherwise the length of $\gamma$ could be reduced by $i_{\alpha+1}-i_{\alpha}-\frac{1}{2}$ in the same homotopy class in $\pi_{1}(M, \operatorname{rel}(p, q))$ as long as $d\left(s_{i_{\alpha+1}}, s_{i_{\alpha}}\right)<\frac{1}{2}$ and then for sufficiently large $n$ (2.26) and (2.27) would imply that $u_{n}$ were not energy minimizing in its homotopy class. A contradiction to the assumption. The group $\pi_{1}(M, \operatorname{rel}(p, q))$ is isomorphic to $\mathbb{Z} \times \ldots \times \mathbb{Z}$, the direct product of finite, say $m$, copies of $\mathbb{Z}$. For any $i_{\alpha_{1}}<i_{\alpha_{2}}<i_{\alpha_{3}}$, there are integers $a_{j}, b_{j}, c_{j}$ for $j=1, \ldots, m$ such that

$$
\begin{aligned}
& {\left[\gamma\left(s_{i_{\alpha_{1}}}, s_{i_{\alpha_{2}}}\right)\right]=\left(a_{1}, \ldots, a_{m}\right) \in \pi_{1}(M, \operatorname{rel}(p, q))} \\
& {\left[\gamma\left(s_{i_{\alpha_{2}}}, s_{i_{\alpha_{3}}}\right)\right]=\left(b_{1}, \ldots, b_{m}\right) \in \pi_{1}(M, \operatorname{rel}(p, q))} \\
& {\left[\gamma\left(s_{i_{\alpha_{1}}}, s_{i_{\alpha_{2}}}\right) \cup \gamma\left(s_{i_{\alpha_{2}}}, s_{i_{\alpha_{3}}}\right)\right]=\left(c_{1}, \ldots, c_{m}\right) \in \pi_{1}(M, \operatorname{rel}(p, q)) .}
\end{aligned}
$$

If there is $j, 1 \leq j \leq m$, such that

$$
\left|a_{j}\right|+\left|b_{j}\right|>\left|c_{j}\right|
$$

then we can shorten $\gamma\left(s_{i_{\alpha_{1}}}, s_{i_{\alpha_{3}}}\right)$ in its homotopy class, and this in turn yields a contradiction as before. Now we have ruled out possible cancelation of homotopy along $\gamma(p, q)$. If we denote the homotopy class of $\gamma^{\prime}(p, q) \cup T_{\infty} \backslash \gamma(p, q)$ by [ $\left.\beta\right]$, then the homotopy class of the loop $\gamma(p, q) \cup \gamma^{\prime}(p, q)$ is equal to $\left[u_{0}\right]-[\beta]$. Hence $\left[\gamma(p, q) \cup \gamma^{\prime}(p, q)\right]$ is bounded, since $\left[u_{0}\right]$ is fixed and $[\beta]$ is bounded. Therefore $\left[\gamma^{\prime}(p, q)\right]$ is large since $[\gamma(p, q)]$ is large, here the homotopy is relative to the fixed boundary $\{p, q\}$. If $\epsilon_{n} R_{n} \rightarrow \infty$ as $n$ goes to infinity, we would have the largest one among the absolute values of the components of $[\gamma(p, q)] \in \mathbb{Z} \times \ldots \times \mathbb{Z}$ tending to infinity. This would imply that $\gamma^{\prime}(p, q)$ is also arbitrarily long. But this contradicts to the choice of $\gamma^{\prime}(p, q)$. So we conclude that the length of the geodesic $\gamma$ is finite if $\gamma$ is the only connecting geodesic line. 
Case 2. there are more than one connecting geodesics. We have $u_{n}\left(\mathcal{C}_{i}\right)$ converges to $\gamma_{i}$ for $i=1, \ldots, k$ where $\mathcal{C}_{i}$ 's are the connecting cylinders. We parameterize the cylinder $\mathcal{C}_{i}$ by $\left[0, R_{n}^{i}\right] \times S^{1}$. Assume $\gamma_{1}$ is very long. As before, this means $\epsilon_{n}^{1} R_{n}^{1}$ is very large. Since $M$ is compact there must exist two points $p_{n}^{1}$ and $q_{n}^{1}$ on $u_{n}\left(\mathcal{C}_{1}\right)$ so that the length the shortest geodesic $\gamma^{\prime}\left(p_{n}^{1}, q_{n}^{1}\right)$ from $p_{n}^{1}$ and $q_{n}^{1}$ in $M$ is very small comparing to $\epsilon_{n}^{1} R_{n}^{1}$. By taking a subsequence if necessary, we can assume $\lim _{n \rightarrow \infty} p_{n}^{1}=p_{1}$ and $\lim _{n \rightarrow \infty} q_{n}^{1}=q_{1}$ where $p_{1}, q_{1} \in \gamma_{1}$. As in Case 1, we conclude that the homotopy class of $\gamma_{1}\left(p_{1}, q_{1}\right)$ must be very large. Although the total homotopy is fixed and equals to $\left[u_{0}\right]$, we cannot draw conclusion as in Case 1 , since the cancelation of the large homotopy of $\gamma_{1}\left(p_{1}, q_{1}\right)$ may comes from other connecting geodesics. Assume $\gamma_{2}, \ldots, \gamma_{s}$ for some $s \leq k$ are the geodesics such that

$$
[\sigma]=\left[\gamma_{1}\left(p_{1}, q_{1}\right)\right]+\left[\gamma_{2}\left(p_{2}, q_{2}\right)\right]+\ldots+\left[\gamma_{s}\left(p_{s} . q_{s}\right)\right]
$$

is bounded. Without loss of generality, we may assume that $\gamma_{i}$ 's are all very long for $i=2, \ldots, s$. There exist shortest geodesics $\gamma_{i}^{\prime}\left(p_{i}, q_{i}\right)$ from $p_{i}$ to $q_{i}$ in $M$ for $i=1, \ldots s$ in the homotopy class $-[\sigma]$. In particular,

$$
\sum_{i=1}^{s} L\left(\gamma_{i}^{\prime}\left(p_{i}, q_{i}\right)\right)
$$

is bounded since $[\sigma]$ is bounded where $L\left(\gamma_{i}^{\prime}\left(p_{i}, q_{i}\right)\right)$ is the length of $\gamma_{i}^{\prime}\left(p_{i}, q_{i}\right)$. Now for $i=1, \ldots, s$, we replace the cylinder $u_{n}\left(\mathcal{C}_{i}\right)$ from $p_{n}^{i}$ to $q_{n}^{i}$, denoted by $u_{n}\left(\mathcal{C}_{i}\left(p_{n}^{i}, q_{n}^{i}\right)\right)$, by the geodesic $\gamma^{\prime}\left(p_{i}, q_{i}\right)$ and identify the boundary components $\partial \mathcal{C}_{i}\left(p_{n}^{i}, q_{n}^{i}\right)$ at $p_{n}^{i}$ and $q_{n}^{i}$ to $p_{n}^{i}$ and $q_{n}^{i}$ respectively. More precisely, we can define comparison maps

$$
u_{n}^{\prime}= \begin{cases}u_{n} & \text { on } \Sigma \backslash \cup_{i=1}^{s} \mathcal{C}_{i}, \\ \gamma^{\prime}\left(p_{i}, q_{i}\right) & \text { on } \cup_{i=1}^{s} \mathcal{C}_{i}\left(p_{n}^{i}, q_{n}^{i}\right),\end{cases}
$$

and on $\left.\cup_{i=1}^{s}\left(\mathcal{C}_{i}\right) \backslash\left(\mathcal{C}_{i}\left(p_{n}^{i}, q_{n}^{i}\right)\right)\right), u_{n}^{\prime}$ is defined by shrinking $u_{n}\left(\partial \mathcal{C}_{i}\left(p_{n}^{i}, q_{n}^{i}\right)\right)$ to $p_{i}, q_{i}$ respectively for each $i=, 1 \ldots, s$. Note that for sufficiently large $n, u_{n}\left(\mathcal{C}_{i}\right)$ converges pointwise to the geodesic $\gamma_{i}$. Hence, in this replacement process, the change in homotopy is zero, and $u_{n}^{\prime}$ is homotopic to $u_{n}$. Further, if $\mathcal{C}_{i}$ is parameterized by $\left[0, R_{n}^{i}\right] \times \mathbb{S}^{1}$ with speed of $u_{n}^{\prime}$ close to that of $u_{n}$, which is close to $\epsilon_{n}^{i}$, we can arrange $u_{n}^{\prime}$ to satisfy

$$
\epsilon_{n}^{i}-C \delta_{1} e^{-\frac{C}{\epsilon_{n}^{i}}} \leq\left|\frac{\partial u_{n}^{\prime}}{\partial t}\right| \leq \epsilon_{n}^{i}+C \delta_{1} e^{-\frac{C}{\epsilon_{n}^{i}}}
$$

and on the cylinder $\mathcal{C}_{i}$

$$
\left|\frac{\partial u_{n}^{\prime}}{\partial \theta}\right| \leq C \delta_{1} e^{-\frac{C}{\epsilon_{n}^{i}}}
$$

where the constant $C$ may be different from those in the estimates for $u_{n}$. As before we have the following estimates for the competing map $u_{n}^{\prime}$ over $\mathcal{C}_{i}$

$$
E\left(u_{n}^{\prime}, \mathcal{C}_{i}\right)=\frac{1}{4}\left(\epsilon_{n}^{i}\right)^{2}{R^{\prime}}_{n}^{i}+O\left(\delta_{1}^{2} e^{-\frac{2 C}{\epsilon_{n}^{i}}}+\delta_{1} \epsilon_{n}^{i} e^{-\frac{C}{\epsilon_{n}^{i}}}\right){R^{\prime}}_{n}^{i}
$$




$$
L\left(u_{n}^{\prime}\left(\mathcal{C}_{i}\right)\right)=\frac{1}{4} \epsilon_{n}^{i} R_{n}^{\prime i}+O\left(\delta_{1} \epsilon_{n}^{i} e^{-\frac{C}{\epsilon_{n}^{i}}}\right) R_{n}^{\prime i} .
$$

But the way the surgery was done implies

$$
L\left(u_{n}^{\prime}\left(\mathcal{C}_{i}\right)\right)<L\left(u_{n}\left(\mathcal{C}_{i}\right)\right)+C_{0}
$$

for some fixed constant $C_{0}>0$ and all $n>n_{0}$ for some fixed $n_{0}$. It follows immediately that there is $n_{0}^{\prime}>0$ such that for all $n>\min \left\{n_{0}, n_{0}^{\prime}\right\}$

$$
E\left(u_{n}^{\prime}, \mathcal{C}\right)<E\left(u_{n}, \mathcal{C}\right)
$$

This contradicts to $u_{n}$ is energy minimizing in the fixed homotopy class with given conformal structure on domain. Therefore we conclude that the length of $\gamma_{1}$ is finite at the first place. We now complete the proof of Proposition 2.7.

Note that the length of a connecting geodesic is bounded by $C \epsilon_{n} R_{n}$ and the energy of $u_{n}$ is bounded by $C \epsilon_{n}^{2} R_{n}$ for some uniform constant $C$, where $R_{n} \rightarrow$ $\infty$ and $\epsilon_{n} \rightarrow 0$ as $n \rightarrow \infty$. Therefore, the finiteness of the geodesics implies immediately that there is no loss of energy on the connecting cylinders in the limit process. This conclusion also holds for the minimizers of the perturbed energy functional in the next section.

In fact, the geodesics arise only from the change of conformal structures, not from the bubbles of harmonic maps. This was proved by Parker in $[\mathrm{P}]$ for a sequence of harmonic maps and Qing-Tian in [QT] for maps with $L^{2}$-bounded tension fields. We only mention some keys points. Recall the Hopf quadratic differential for a map $u$ from a surface is given by

$$
\Phi(u)=\phi(u) d z^{2}
$$

where

$$
\phi(u)=\left|u_{x}\right|^{2}-\left|u_{y}\right|^{2}-2 \sqrt{-1} u_{x} \cdot u_{y}
$$

It is well known that if $u$ is harmonic, then $\Phi(u)$ is holomorphic, namely,

$$
\bar{\partial} \phi(u)=0
$$

The bubbles arising from the convergence of the harmonic maps $u_{n}$ form bubble trees. By a bubble tree, we mean finitely many $\mathbb{S}^{2}$ 's connected by thin cylinders of finite length, and with a half cylinder connect one 2-sphere to a principal component. Before reaching the final limit $u_{\infty}$, each bubble tree is topologically a disk. The $L^{1}$-norm of the Hopf differential is conformally invariant. It follows that

$$
\phi\left(u_{n}\right)=\left|\left(u_{n}\right)_{t}\right|^{2}-\left|\left(u_{n}\right)_{\theta}\right|^{2}-2 \sqrt{-1}\left(u_{n}\right)_{t} \cdot\left(u_{n}\right)_{\theta}
$$

has small $L^{1}\left(D_{r}\right)$-norm if $r$ is small ([DT]). But $\left|\left(u_{n}\right)_{\theta}\right|$ decays exponentially in cylindrical coordinates. Hence, the $L^{1}$-norm of $\left|\left(u_{n}\right)_{t}\right|$ also decays exponentially 
in $t$. So after passing to limit, the length of geodesics connecting the bubbles is zero.

It remains to analyze the local structure near singularities at the nodes on $\Sigma_{\infty}=\left(\Sigma, c_{\infty}\right)$. We need two steps for scaling of metrics: first we scale the metrics $g_{n}$ on $\Sigma$ near the points which will eventually develop cusps as $n \rightarrow \infty$ to make the geometry uniform; second we scale the new metrics near the points where energy concentrates. The analysis involved in the second step is similar to that for the necks which connect the bubbles.

As $n$ approaching to infinity, $\Sigma_{n}$ may develop some regions which eventually converge to cusps of $\Sigma_{\infty}$ (see definition in Section 4). We normalize the metrics $g_{n}$ by conformal changes such that the curvature is everywhere bounded on $\Sigma$ and there is a uniform lower bound on the injectivity radius with respect to the new metrics $\tilde{g_{n}}$ for all $n$. Notice that the Dirichlet energy and harmonicity of maps are invariant under conformal transformations on $\Sigma$. Therefore, in metrics $\tilde{g}_{n}, u_{n}$ is energy minimizing in the given homotopy class, $E\left(u_{n}\right)$ is uniformly bounded above and on $\left(\Sigma, \tilde{g}_{n}\right)$ the injectivity radius is uniformly bounded below and the curvature is uniformly bounded. As before, there are points $x_{n 1}, \ldots, x_{n m}$ and such that for any point $x \notin \cup_{i=1}^{m} B_{2}\left(x_{n i}\right)$,

$$
\int_{B_{1}(x)}\left|\nabla u_{n}\right|_{\tilde{g_{n}}}^{2} d A_{\tilde{g_{n}}} \leq C \epsilon
$$

Note that $m$ is independent of $n$. The $\epsilon$-regularity theorem then asserts

$$
\left\|u_{n}\right\|_{C^{2}\left(B_{\frac{1}{2}}(x)\right)} \leq C \sqrt{\epsilon}
$$

Now we turn our attention to the unit balls, measured in the metric $\tilde{g_{n}}$, centered in $\cup_{i=1}^{m} B_{2}\left(x_{n i}\right)$. These are the regions where the Dirichlet energy concentrated. For simplicity, we assume that $m=1$ and the general case can be handled in the same way. Rescale the metric $\tilde{g}_{n}$ on the unit ball such that $\left\|\nabla u_{n}\right\|_{C^{0}} \leq 1$. After rescaling, $\left\|u_{n}\right\|_{C^{2}} \leq C \sqrt{\epsilon}$ everywhere. By a selection of subsequence from $\left\{u_{n}\right\}$ and we still use $\left\{u_{n}\right\}$ to denote it, $\left\{u_{n}\right\}$ converge to a harmonic map $u_{\infty}$ from $\Sigma_{\infty} \backslash\left\{x_{\infty 1}, \ldots, x_{\infty m}\right\}$ union with $m 2$-spheres $\mathbb{S}_{i}^{2}$ into $M$. Next, according to the removable singularity theorem, $u_{\infty}$ extends to a harmonic map from $\Sigma_{\infty} \cup_{i=1}^{m} \mathbb{S}_{i}^{2}$. Note that harmonic maps from $\mathbb{S}^{2}$ are conformal. Finally, since after rescaling, the energy density of $u_{n}$ is small pointwise. The image of long cylinders converges to geodesics of finite length as before.

Finally, we maps the 1-dimensional component of the stratified Riemann surfaces into geodesics in $M$ with finite length. Now the proof of Theorem 1.1 is completed. 


\section{Harmonic nodal maps as limit of critical points of Sacks- Uhlenbeck's functional}

In order to study the existence problem, we will investigate a broader class of elliptic equations, namely the critical point of the perturbed energy functional. We point out that in order to prove Theorem 1.1, we only need to consider harmonic maps rather than the perturbed energy, on the other hand, to conclude the existence of harmonic maps, we only need to deal with the perturbed energy with fixed domain metric.

We first recall the perturbed energy functional introduced in [SU1]. Let $\Sigma$ be a compact Riemann surface with a metric $g$. For any $n$ and a constant $\beta>1$ and a map $u: \Sigma \rightarrow M$, define

$$
E_{\beta}(u)=\frac{1}{2} \int_{\Sigma}\left(1+|\nabla u|^{2}\right)^{\beta} d A-\frac{1}{2} \operatorname{Area}(\Sigma) .
$$

Since $\beta>1$, it is clear that

$$
E_{\beta}(u) \geq E(u)
$$

where $E(u)$ is the Dirichlet energy of $u$ with respect to the metric $g$. The EulerLagrange equation for the functional $E_{\beta}$ is

$$
\triangle u^{k}+\frac{(\beta-1) \sum_{j=1}^{2} \nabla_{j} u^{k} \nabla_{j}|\nabla u|^{2}}{1+|\nabla u|^{2}}=0
$$

for $k=1, \ldots, \operatorname{dim} M$ where

$$
\triangle u^{k}=g^{s t} \partial_{s t}^{2} u^{k}-g^{s t} \Gamma_{i j}^{k}(u) \partial_{i} u^{s} \partial_{j} u^{t}
$$

and $\Gamma_{i j}^{k}$ 's are the Christoffel symbols of $(M, h)$.

Lemma 3.1. For each $u_{n}$ and $\beta_{n}>1$, there exists a minimizer for the functional $E_{\beta_{n}}$ among mappings which are homotopic to $u_{n}$.

Proof. Take an $E_{\beta_{n}}$-minimizing sequence $\left\{v_{n i}\right\}$ in the homotopy class determined by $u_{n}$. For any point $x \in \Sigma$,

$$
\begin{aligned}
\int_{B_{\delta}(x)}\left|\nabla v_{n i}\right|_{g}^{2} d A_{g} & \leq\left(\int_{B_{\delta}(x)}\left|\nabla v_{n i}\right|^{2 \beta_{n}}\right)^{\frac{1}{\beta_{n}}} \operatorname{Area}\left(B_{\delta}(x)\right)^{\frac{\beta_{n}-1}{\beta_{n}}} \\
& \leq C \delta^{\frac{2 \beta_{n}-2}{\beta_{n}}}
\end{aligned}
$$

by Hölder inequality. Therefore, Courant-Lebesque lemma (cf. [J1]) implies that $\left\{v_{n i}\right\}$ is equicontinuous. A subsequence of $\left\{v_{n i}\right\}$ converge to a map $v_{n}$, by ArzelaAscoli's theorem, and $v_{n}$ is Lipschitz continuous hence smooth. So $v_{n}$ is a $E_{\beta_{n}}$ minimizer which is homotopic to $u_{n}$. 
Let $T$ be a stratified Riemann surface associated to $\Sigma$. Since $u_{0} \in C^{\infty}(T, M)$, it has finite energy. Set

$$
\alpha=\inf \left\{E(u) \mid[u]=\left[u_{0}\right]\right\} .
$$

If $\alpha=0$, a constant map is in the homotopy class $\left[u_{0}\right]$ which is clearly a harmonic nodal map. Assume $\alpha>0$. Take an energy minimizing sequence $\left\{v_{n}\right\}$ in $C^{\infty}(T, M)$ such that $\left[v_{n}\right]=\left[u_{0}\right]$ and

$$
\lim _{n \rightarrow \infty} E\left(v_{n}\right)=\alpha .
$$

We may assume without loss of any generality that $T$ has only one principal component, since we can always construct a minimizer on each principal component then take the union of them. For the fixed domain metric $g$, we will improve the minimizing sequence $\left\{v_{n}\right\}$ within the given homotopy class, so that the new minimizing sequence $\left\{u_{n}\right\}$ consists of critical points of the perturbed energy functional. To do so, we pick a sequence of numbers $\left\{\beta_{n}\right\}$ satisfying (1) $\beta_{n}>1$ and (2) $\lim _{n \rightarrow \infty} \beta_{n}=1$. For each $\beta_{n}$, let $u_{n}$ be an $E_{\beta_{n}}$-minimizer in the homotopy class $\left[v_{n}\right]=\left[u_{0}\right]$. Moreover, as $\beta_{n}$ tends to 1 , there exists a sequence of positive numbers $\left\{\epsilon_{n}\right\}$ with $\lim _{n \rightarrow \infty} \epsilon_{n}=0$ such that

$$
E_{\beta_{n}}\left(u_{n}\right) \leq \alpha+\epsilon_{n}
$$

because $\left\{v_{n}\right\}$ is a minimizing sequence and the $E_{\beta_{n}}$-energy approaches to the Dirichlet energy as $\beta_{n}$ goes to 1 . So $\left\{u_{n}\right\}$ is indeed a minimizing sequence in the given homotopy class.

Note that both the $\epsilon$-regularity theorem and the removable singularity theorem remain true for $E_{\beta}$-minimizers if $\beta>1$. Therefore, the blowing up process discussed in Section 2 still works for the minimizers $u_{n}$. In particular, there are finitely many points $x_{n 1}, \ldots, x_{n m}$ in $\Sigma$, where $m$ is independent of $n$ and $\beta_{n}$, such that for any $x \notin \cup_{i=1}^{m} B_{2}\left(x_{n i}\right)$,

$$
\int_{B_{1}(x)}\left|\nabla u_{n}\right|_{g}^{2} d A_{g} \leq C \epsilon
$$

and for any $i=1, \ldots, m$

$$
\lim _{n \rightarrow \infty} \max _{y \in B_{1}\left(x_{n i}, g\right)}\left|\nabla u_{n}\right|(y)=\infty .
$$

Then we rescale the metric as in Section 2 and observe that this rescaling process stops after finitely many times. Then by Courant-Lebesque lemma (cf. [J1]), a subsequence of $\left\{u_{n}\right\}$ converges to a harmonic map as $n \rightarrow \infty, \beta \rightarrow 1$ from $\Sigma$ union with finitely many 2 -spheres. 
In the sequel, we will use $u, \tilde{u}$ and $\beta$ for $u_{n}, \tilde{u_{n}}$ and $\beta_{n}$ respectively when there is no ambiguity. Along the annulus we have a map $u$ into $M$ satisfying (3.3). By the conformal transformation $(t, \theta) \rightarrow(-\log t, \theta)$, we have

$$
\left\{\begin{array}{l}
|\nabla u|^{2}=e^{2 t}\left(\left|u_{t}\right|^{2}+\left|u_{\theta}\right|^{2}\right) \\
\triangle u=e^{2 t}\left(u_{t t}+u_{\theta \theta}\right)
\end{array}\right.
$$

where $|\cdot|$ is the length in $R^{N}$ and the second derivatives are covariant differentiation. After rescaling the metric

$$
\left\{\begin{array}{l}
\|\nabla u\|_{C^{0}} \leq C \\
\|\nabla u\|_{C^{0}(C)} \leq \delta
\end{array}\right.
$$

for some $\beta>1$ and some small positive constant $\delta$. It follows that $u$ satisfies the following differential equation on the cylinder $\mathcal{C}=[0, R] \times \mathbb{S}^{1}$. In the sequel, "." stands for the inner product of two vectors.

$$
\begin{aligned}
& u_{t t}+u_{\theta \theta} \\
& +2(\beta-1) \frac{u_{t}\left(\left|u_{t}\right|^{2}+\left|u_{\theta}\right|^{2}\right)+u_{t} u_{t t} \cdot u_{t}+u_{\theta} u_{\theta \theta} \cdot u_{\theta}+u_{\theta} u_{t} \cdot u_{t \theta}+u_{t} u_{\theta} \cdot u_{t \theta}}{e^{-2 t}+\left|u_{t}\right|^{2}+\left|u_{\theta}\right|^{2}} \\
& =0 .
\end{aligned}
$$

Let $\tilde{u}$ be the $\theta$-independent solution of (3.7). Then

$$
\tilde{u}_{t t}^{k}+\frac{2(\beta-1) \tilde{u}_{t}^{k} \sum_{j} \tilde{u}_{t}^{j} \tilde{u}_{t t}^{j}+2(\beta-1) \tilde{u}_{t}^{k}\left|\tilde{u}_{t}\right|^{2}}{e^{-2 t}+\left|\tilde{u}_{t}\right|^{2}}=0
$$

for $k=1, \ldots \operatorname{dim} M$ where $\tilde{u}_{t t}$ is the nonlinear Laplacian of $\tilde{u}$.

Lemma 3.2. In the cylindrical coordinate $(t, \theta)$, there is a unique solution $\tilde{u}$ of (3.8) for $t \in[0, R]$ over $\mathcal{C}$ for given initial data.

Proof. Set $w=\tilde{u}_{t}$. Then by (3.8)

$$
w_{t}^{k}=-\frac{(\beta-1) w^{k} \sum_{j} w^{j} w_{t}^{j}+2(\beta-1) w^{k}|w|^{2}}{e^{-2 t}+|w|^{2}} .
$$

Since $\beta$ is sufficiently close to 1 , the right hand side of the above equation has the form

$$
\sum_{j} \epsilon_{1}^{j} w_{t}^{j}+\epsilon_{2} w
$$

where $\epsilon_{1}$ and $\epsilon_{2}$ depend on $w$ but their $C^{0}$-norms are close to 0 . Then it is clear that $w$ exists for long time, which in turn implies by integration that $\tilde{u}$ has long time existence. 
Lemma 3.3. Let $\tilde{u}$ be a solution of (3.8). Then

(i) $\left|\tilde{u}_{t}\right|$ is decreasing in $t$;

(ii) for any $t \leq t_{0}$,

$$
\left|\tilde{u}_{t}\left(t_{0}\right)\right|^{2} e^{-\frac{4(\beta-1)}{2 \beta-1}\left(t-t_{0}\right)}-\frac{1}{2 \beta-1} e^{-2 t} \leq\left|\tilde{u}_{t}(t)\right|^{2} \leq\left|\tilde{u}_{t}\left(t_{0}\right)\right|^{2} e^{-\frac{4(\beta-1)}{2 \beta-1}\left(t-t_{0}\right)} .
$$

Proof. We set

$$
f(t)=\left|\tilde{u}_{t}(t)\right|^{2} .
$$

Then multiplying $\tilde{u}_{t}^{k}$ on (3.8) and summing over $k$ yields

$$
f_{t}+2(\beta-1) \frac{f f_{t}+2 f^{2}}{e^{-2 t}+f}=0 .
$$

Rearrangement of (3.11) leads to

$$
f_{t}=-\frac{4(\beta-1) f^{2}}{e^{-2 t}+(2 \beta-1) f} .
$$

Now (i) follows immediately from (3.12). As for (ii), we first observe that

$$
f_{t} \geq \frac{4(\beta-1)}{2 \beta-1} f
$$

Integrate (3.13) from $t_{0}$ to $t$ and notice that $t \leq t_{0}$. So the first inequality in (3.9) holds. On the other hand, to derive the second inequality, we let

$$
v=f+\frac{1}{2 \beta-1} e^{-2 t}
$$

Then by direct computation, we have

$$
\begin{aligned}
-v_{t} & =-f_{t}+\frac{2}{2 \beta-1} e^{-2 t} \\
& =\frac{4(\beta-1)}{2 \beta-1} \frac{v^{2}-\frac{2}{2 \beta-1} v e^{-2 t}+\frac{1}{(2 \beta-1)^{2}} e^{-4 t}}{v}+\frac{2}{2 \beta-1} e^{-2 t} \\
& =\frac{4(\beta-1)}{2 \beta-1} v+\left(\frac{2}{2 \beta-1}-\frac{8(\beta-1)}{(2 \beta-1)^{2}}\right) e^{-2 t}+\frac{4(\beta-1)}{(2 \beta-1)^{3}} e^{-4 t}
\end{aligned}
$$

It follows that

$$
-v_{t} \geq \frac{4(\beta-1)}{2 \beta-1} v
$$


Since $t \leq t_{0},(3.16)$ implies that

$$
\log v\left(t_{0}\right)-\log v(t) \leq \frac{4(\beta-1)}{2 \beta-1}\left(t-t_{0}\right) .
$$

Therefore due to (3.17) and (3.14),

$$
f(t)+\frac{1}{2 \beta-1} e^{-2 t} \geq\left(f\left(t_{0}\right)+\frac{1}{2 \beta-1} e^{-2 t_{0}}\right) e^{-\frac{4(\beta-1)}{2 \beta-1}\left(t-t_{0}\right)}
$$

Now the second inequality follows.

Lemma 3.4. Let $\tilde{u}$ be a solution of (3.8). Then for $\beta$ sufficiently close to 1 , there is a uniform positive constant $C$ such that

$$
\left|\tilde{u}_{t t}\right| \leq C(\beta-1)\left|\tilde{u}_{t}\right| .
$$

Proof. By (3.8) and the triangle inequality,

$$
\left|\tilde{u}_{t t}\right|-2(\beta-1) \frac{\left|\tilde{u}_{t}\right|^{2}}{e^{-2 t}+\left|\tilde{u}_{t}\right|^{2}}\left|\tilde{u}_{t t}\right| \leq 2(\beta-1)\left|\tilde{u}_{t}\right| .
$$

Since $\beta-1$ is small, (3.19) follows from (3.20).

Now we derive the equation for

$$
w=u-\tilde{u} .
$$

In order to apply Corollary 2.4 , we need to show that $w$ satisfies a P.D.E of the form (2.11) which satisfying (2.12) and (2.13). First, we notice that since $\tilde{u}$ is $\theta$-independent,

$$
\frac{\left|u_{\theta}\right|^{2} u_{t}^{k}+u_{\theta} \cdot u_{\theta \theta} u_{\theta}^{k}+u_{\theta} \cdot u_{t \theta} u_{t}^{k}+u_{t} \cdot u_{t \theta} u_{\theta}^{k}}{e^{-2 t}+\left|u_{t}\right|^{2}+\left|u_{\theta}\right|^{2}}=A^{i \theta} D_{i \theta}^{2} w+B \cdot w_{\theta}
$$

where for some uniform constant $C$,

$$
\|A\|_{C^{0}}+\|B\|_{C^{0}} \leq C
$$

Secondly, we have

$$
\begin{aligned}
I_{1} & :=\frac{u_{t}^{k}\left|u_{t}\right|^{2}}{e^{-2 t}+\left|u_{t}\right|^{2}+\left|u_{\theta}\right|^{2}}-\frac{\tilde{u}_{t}^{k}\left|\tilde{u}_{t}\right|^{2}}{e^{-2 t}+\left|\tilde{u}_{t}\right|^{2}} \\
& =\frac{\left|u_{t}\right|^{2}}{e^{-2 t}+\left|u_{t}\right|^{2}+\left|u_{\theta}\right|^{2}} w_{t}^{k}+\tilde{u}_{t}^{k}\left(\frac{\sum_{j} e^{-2 t}\left(u_{t}^{j}+\tilde{u}_{t}^{j}\right) w_{t}^{j}-\left|u_{\theta}\right|^{2}\left|\tilde{u}_{t}\right|^{2}}{\left(e^{-2 t}+\left|u_{t}\right|^{2}+\left|u_{\theta}\right|^{2}\right)\left(e^{-2 t}+\left|\tilde{u}_{t}\right|^{2}\right)}\right)
\end{aligned}
$$


For the last term in (3.24),

$$
\begin{aligned}
\frac{\tilde{u}_{t}^{k}\left|\tilde{u}_{t}\right|^{2}\left|u_{\theta}\right|^{2}}{\left(e^{-2 t}+\left|u_{t}\right|^{2}+\left|\tilde{u}_{t}\right|^{2}\right)\left(e^{-2 t}+\left|\tilde{u}_{t}\right|^{2}\right)} & =-\frac{\left|u_{\theta}\right|^{2}\left|\tilde{u}_{t}\right|^{2} w_{t}^{k}}{\left(e^{-2 t}+\left|u_{t}\right|^{2}+\left|\tilde{u}_{t}\right|^{2}\right)\left(e^{-2 t}+\left|\tilde{u}_{t}\right|^{2}\right)} \\
& +\frac{\sum_{j} u_{t}^{k} u_{\theta}^{j}\left|\tilde{u}_{t}\right|^{2} w_{\theta}^{j}}{\left(e^{-2 t}+\left|u_{t}\right|^{2}+\left|\tilde{u}_{t}\right|^{2}\right)\left(e^{-2 t}+\left|\tilde{u}_{t}\right|^{2}\right)} .
\end{aligned}
$$

Combining (3.24) and (3.25) together, we see that

$$
I_{1}=V \cdot \nabla w
$$

where $C$ is some uniform positive constant and $V$ is a $\mathbb{R}^{N}$-valued function which satisfies

$$
\|V\|_{C^{0}} \leq C .
$$

Finally, for the terms involving $u_{t t}$ and $\tilde{u}_{t t}$, we have

$$
\begin{aligned}
I_{2} & :=\frac{\sum_{j} u_{t}^{j} u_{t t}^{j} u_{t}^{k}}{e^{-2 t}+\left|u_{t}\right|^{2}+\left|u_{\theta}\right|^{2}}-\frac{\sum_{j} \tilde{u}_{t}^{j} \tilde{u}_{t t}^{j} \tilde{u}_{t}^{k}}{e^{-2 t}+\left|\tilde{u}_{t}\right|^{2}} \\
& =\frac{\sum_{j} u_{t}^{j} w_{t t}^{j} u_{t}^{k}}{e^{-2 t}+\left|u_{t}\right|^{2}+\left|u_{\theta}\right|^{2}}+\frac{\sum_{j} u_{t}^{j} \tilde{u}_{t t}^{j} u_{t}^{k}}{e^{-2 t}+\left|u_{t}\right|^{2}+\left|u_{\theta}\right|^{2}} \\
& -\frac{\sum_{j} \tilde{u}_{t}^{j} \tilde{u}_{t t}^{j} u_{t}^{k}}{e^{-2 t}+\left|\tilde{u}_{t}\right|^{2}}-\frac{\sum_{j} \tilde{u}_{t}^{j} \tilde{u}_{t t}^{j} w_{t}^{k}}{e^{-2 t}+\left|\tilde{u}_{t}\right|^{2}} .
\end{aligned}
$$

We denote the two terms in the middle of the right hand side of (3.28) by $J$. Therefore

$$
\begin{aligned}
J & =\sum_{j} \tilde{u}_{t t}^{j} u_{t}^{k}\left(\frac{u_{t}^{j}}{e^{-2 t}+\left|u_{t}\right|^{2}+\left|u_{\theta}\right|^{2}}-\frac{\tilde{u}_{t}^{j}}{e^{-2 t}+\left|\tilde{u}_{t}\right|^{2}}\right) \\
& =\sum_{j} \tilde{u}_{t t}^{j} u_{t}^{k}\left(\frac{e^{-2 t}\left(u_{t}^{j}-\tilde{u}_{t}^{j}\right)}{\left(e^{-2 t}+\left|u_{t}\right|^{2}+\left|u_{\theta}\right|^{2}\right)\left(e^{-2 t}+\left|\tilde{u}_{t}\right|^{2}\right)}\right) \\
& -\sum_{j} \tilde{u}_{t t}^{j} u_{t}^{k}\left(\sum_{l} \frac{\tilde{u}_{t}^{j} u_{\theta}^{l}\left(u_{\theta}^{l}-\tilde{u}_{\theta}^{l}\right)}{\left(e^{-2 t}+\left|u_{t}\right|^{2}+\left|u_{\theta}\right|^{2}\right)\left(e^{-2 t}+\left|\tilde{u}_{t}\right|^{2}\right)}\right) \\
& +\sum_{j} \tilde{u}_{t t}^{j} u_{t}^{k}\left(\frac{u_{t}^{j}\left|\tilde{u}_{t}\right|^{2}-\tilde{u}_{t}^{j}\left|u_{t}\right|^{2}}{\left(e^{-2 t}+\left|u_{t}\right|^{2}+\left|u_{\theta}\right|^{2}\right)\left(e^{-2 t}+\left|\tilde{u}_{t}\right|^{2}\right)}\right) .
\end{aligned}
$$

According to (3.19) in Lemma 3.4, the first two terms in (3.29) consist of $\nabla w$ times a matrix with bounded entries. As for the last term in (3.29), we have

$$
\begin{aligned}
u_{t}^{j} \tilde{u}_{t}^{l} \tilde{u}_{t}^{l}-\tilde{u}_{t}^{j} u_{t}^{l} u_{t}^{l} & =u_{t}^{j} \tilde{u}_{t}^{l}\left(\tilde{u}_{t}^{l}-u_{t}^{l}\right)+\left(u_{t}^{j} \tilde{u}_{t}^{l}-\tilde{u}_{t}^{j} u_{t}^{l}\right) u_{t}^{l} \\
& =-u_{t}^{j} \tilde{u}_{t}^{l} w_{t}^{l}+w_{t}^{j} \tilde{u}_{t}^{l} u_{t}^{l}+\tilde{u}_{t}^{j} w_{t}^{l} u_{t}^{l} .
\end{aligned}
$$


So again by (3.19), the last term in (3.29) is also in the form of $\nabla w$ times a matrix with bounded entries. Therefore we conclude that

$$
I_{2}=E w_{t t}+F \nabla w
$$

where the $C^{0}$-norms of $E$ and $F$ are uniformly bounded. Putting (3.21), (3.26) and (3.31) together, we obtain the following equation for $u-\tilde{u}$ :

$$
w_{t t}+w_{\theta \theta}+(\beta-1) A D^{2} w+(\beta-1) B \nabla w=0
$$

where for some uniform constant $C$,

$$
\|A\|_{C^{0}}+\|B\|_{C^{0}} \leq C
$$

Since the image space $M$ may not be flat, the second derivatives in (3.33) involve the Levi-Civita connection of $M$. The nonlinear terms are

$$
\Gamma_{j k}^{i}(u) u_{\alpha}^{j} u_{\beta}^{k}-\Gamma_{j k}^{i}(\tilde{u}) \tilde{u}_{\alpha}^{j} \tilde{u}_{\beta}^{k}
$$

where $\Gamma_{j k}^{i}$ 's are the Christofell symbols on $M$. (3.34) can be written as

$$
A_{1} \nabla w+A_{2} w
$$

where since $M$ is compact, there is a uniform constant $C$ such that

$$
\left\{\begin{array}{l}
\left|A_{1}\right| \leq C(|\nabla u|+|\nabla \tilde{u}|) \\
\left|A_{2}\right| \leq C\left(|\nabla u|^{2}+|\nabla \tilde{u}|^{2}\right)
\end{array}\right.
$$

But $\beta-1$ is sufficiently small, and therefore $w$ satisfies a homogeneous equation in the form of (2.11) with all the conditions in Corollary 2.4 hold.

Proposition 3.5. Let $u$ and $\tilde{u}$ be solutions of (3.7) and (3.8) respectively along $\mathcal{C}=[0, R] \times \mathbb{S}^{1}$. Let $\delta$ be the positive number $\delta_{1}$ in Corollary 2.4. Assume that on $\mathcal{C}$

$$
|\nabla u| \leq \epsilon<\frac{\delta}{8}
$$

If $u$ and $\tilde{u}$ satisfy the normalization conditions (2.16) and (2.17), then

$$
\left|\tilde{u}_{t}\right| \leq \delta
$$

along $\mathcal{C}$.

Proof. Since $u-\tilde{u}$ satisfies (2.11) and the normalization conditions (2.16), (2.17), by Corollary 2.4, we can choose $t_{0}=i_{0} l$ such that

$$
i_{0}=\min _{i}\left\{i \mid(a)\|u-\tilde{u}\|_{i} \leq e^{-\frac{l}{2}}\|u-\tilde{u}\|_{i-1},(b)\|u-\tilde{u}\|_{i} \leq e^{-\frac{l}{2}}\|u-\tilde{u}\|_{i+1}\right\} .
$$


On $I_{i_{0}}$, by the initial condition we may assume that

$$
\left|\tilde{u}_{t}\right|<2 \epsilon .
$$

Notice that for any $t>t_{0},(3.40)$ holds since $\left|\tilde{u}_{t}\right|$ is decreasing in $t$ by Lemma 3.3. Now we go backward along the cylinder $\mathcal{C}$ from $t_{0}$. If (3.38) were not true, we would meet three points $t_{1}, t_{2}, t_{3}$ such that

$$
\left|\tilde{u}_{t}\right|=\left\{\begin{array}{l}
\frac{1}{4} \delta \text { at } t_{1} \\
\frac{1}{2} \delta \text { at } t_{2} \\
\delta \text { at } t_{3},
\end{array}\right.
$$

and moreover $\left|\tilde{u}_{t}\right|$ didn't take these values before the corresponding points. Clearly,

$$
t_{3}<t_{2}<t_{1}<t_{0} .
$$

Notice that Corollary 2.4 is at least valid on $\left[t_{3}, R\right] \times \mathbb{S}^{1}$. Therefore for any $t \in$ $\left[t_{3}, t_{0}\right]$

$$
|\nabla u-\nabla \tilde{u}| \leq C \delta e^{-\frac{1}{2}\left(t-t_{3}\right)} .
$$

This in turn implies that

$$
\frac{\delta}{4} \leq C \delta e^{-\frac{1}{2}\left(t_{1}-t_{3}\right)}+\epsilon<C \delta e^{-\frac{1}{2}\left(t_{1}-t_{3}\right)}+\frac{1}{8} \delta .
$$

So there is a uniform upper bound for $t_{1}-t_{3}$ :

$$
0 \leq t_{1}-t_{3} \leq-2 \log \frac{1}{8 C} .
$$

On the other hand, according to Lemma 3.3 (take $t_{1}$ to be the $t_{0}$ in Lemma 3.3),

$$
\left|\tilde{u}_{t}\left(t_{2}\right)\right| \leq\left|\tilde{u}_{t}\left(t_{1}\right)\right| e^{-\frac{4(\beta-1)}{2 \beta-1}\left(t_{2}-t_{1}\right)} .
$$

By the way we choose $t_{1}, t_{2}$, we conclude

$$
t_{1}-t_{2} \geq \frac{(2 \beta-1) \log 2}{4(\beta-1)} .
$$

But if $\beta$ is sufficiently close to 1 , (3.47) implies that $t_{1}-t_{3}>t_{1}-t_{2}$ approaches to infinity. This contradicts to the uniform upper bound (3.45) for $t_{1}-t_{3}$.

Therefore, we have shown that Corollary 2.4 can be applied on the entire cylinder $\mathcal{C}$. So we have as in Proposition 2.6 that

$$
\begin{aligned}
& |u-\tilde{u}|+|\nabla u-\nabla \tilde{u}| \leq C \delta e^{-\frac{1}{2} t}, \quad \text { if } t \leq t_{0} \\
& |u-\tilde{u}|+|\nabla u-\nabla \tilde{u}| \leq C \delta e^{-\frac{1}{2}(R-t)}, \quad \text { if } t \geq t_{0} .
\end{aligned}
$$


Next, we reparameterize the $\theta$-independent solution $\tilde{u}$ by its arc length and derive corresponding equation for $\tilde{u}$ in the new parameter. Set

$$
s=\int_{t_{0}}^{t}\left|\tilde{u}_{t}\right| .
$$

In particular, $s$ is increasing in $t$, hence its inverse function exists. We have

$$
\left\{\begin{array}{l}
\tilde{u}_{s}=\tilde{u}_{t} t_{s} \\
\tilde{u}_{s s}=\tilde{u}_{t t} t_{s}^{2}+\tilde{u}_{t} t_{s s}=\tilde{u}_{t t} t_{s}^{2}+\frac{t_{s s}}{t_{s}} \tilde{u}_{s} \\
\left|\tilde{u}_{s}\right|=\left|\frac{\tilde{u}_{t}}{s_{t}}\right|=\left|\frac{\tilde{u}_{t}}{\left|\tilde{u}_{t}\right|}\right|=1 .
\end{array}\right.
$$

Therefore we can use the arc length $s$ to rewrite (3.8) as follows

$$
\frac{1}{t_{s}^{2}}\left(\tilde{u}_{s s}^{k}-\frac{t_{s s}}{t_{s}} \tilde{u}_{s}^{k}\right)-2(\beta-1) \frac{t_{s}^{-1} \tilde{u}_{s}^{k}\left(\left(\tilde{u}_{s s}-t_{s s} t_{s}^{-1} \tilde{u}_{s}\right) \cdot t_{s}^{-3} \tilde{u}_{s}+t_{s}^{-2}\left|\tilde{u}_{s}\right|^{2}\right)}{e^{-2 t}+t_{s}^{-2}\left|\tilde{u}_{s}\right|^{2}}=0 .
$$

Since $\tilde{u}_{s} \cdot \tilde{u}_{s}=1$, it follows by differentiation that

$$
\tilde{u}_{s} \cdot \tilde{u}_{s s}=\sum_{k} \tilde{u}_{s}^{k} \tilde{u}_{s s}^{k}=0
$$

Thus, we can simplify the above equation for $\tilde{u}$ as

$$
\tilde{u}_{s s}^{k}-\tilde{u}_{s}^{k}\left(\frac{t_{s s}}{t_{s}}-2(\beta-1) \frac{t_{s}^{-1}-t_{s s} t_{s}^{-3}}{e^{-2 t}+t_{s}^{-1}}\right)=0 .
$$

Multiplying $\tilde{u}_{s}^{k}$ then summing over $k$, we obtain

$$
\frac{t_{s s}}{t_{s}}-2(\beta-1) \frac{t_{s}^{-1}-t_{s s} t_{s}^{-3}}{e^{-2 t}+t_{s}^{-1}}=0 .
$$

Then (3.54) and (3.55) together implies that

$$
\tilde{u}_{s s}^{k}=0
$$

for every $k$. So we have shown

Proposition 3.6. For the $\theta$-independent solution $\tilde{u}$ of (3.8), if the cylinder is parameterized by the arc length $s$, then $\tilde{u}(s)$ is a geodesic. 
Therefore we have shown that $u$ converges to a geodesic in $C^{0}$-norm. Next, we give an estimate for the energy density of $\theta$-independent solution $\tilde{u}$, which will be useful to study the energy of $u$ along the cylinder.

Proposition 3.7. Let $\tilde{u}$ be a $\theta$-independent solution. Then

$$
\begin{aligned}
& \left.|| \tilde{u}_{t}\right|^{2}-\left|\tilde{u}_{t}\left(\frac{1}{2} R\right)\right|^{2} e^{-\frac{4(\beta-1)}{2 \beta-1}(t-R / 2)} \mid \leq C e^{-C t}, \quad \text { if } t<\frac{R}{2}, \\
& \left.|| \tilde{u}_{t}\right|^{2}-\left|\tilde{u}_{t}\left(\frac{1}{2} R\right)\right|^{2} e^{-\frac{4(\beta-1)}{2 \beta-1}(t-R / 2)} \mid \leq C e^{-C(R-t)}, \text { if } t \geq \frac{R}{2}
\end{aligned}
$$

for some uniform positive constant $C$.

Proof. Let

$$
\tilde{F}=\left|\tilde{u}_{t}\right|^{2}-\left|\tilde{u}_{t}\left(\frac{1}{2} R\right)\right|^{2} e^{-\frac{4(\beta-1)}{2 \beta-1}(t-R / 2)} .
$$

Clearly,

$$
\tilde{F}\left(\frac{1}{2} R\right)=0 .
$$

It's straight forward to obtain

$$
\tilde{F}_{t}=-\frac{4(\beta-1)}{2 \beta-1} \tilde{F}+\frac{4(\beta-1)}{2 \beta-1} \frac{e^{-2 t}\left|\tilde{u}_{t}\right|^{2}}{e^{-2 t}+(2 \beta-1)\left|\tilde{u}_{t}\right|^{2}} .
$$

Therefore

$$
\tilde{F}=\frac{4(\beta-1)}{2 \beta-1} e^{-\frac{4(\beta-1)}{2 \beta-1}(t-R / 2)} \int_{t}^{\frac{R}{2}} e^{\frac{4(\beta-1)}{2 \beta-1}(s-R / 2)} \frac{e^{-2 s}\left|\tilde{u}_{t}\right|^{2}}{e^{-2 s}+(2 \beta-1)\left|\tilde{u}_{t}\right|^{2}} d s .
$$

If $t<\frac{R}{2}$, then

$$
\begin{aligned}
|\tilde{F}| & \leq \frac{4(\beta-1)}{2 \beta-1} \int_{t}^{\frac{R}{2}} e^{\frac{4(\beta-1)}{2 \beta-1}(s-t)} e^{-2 s} d s \\
& \leq C \frac{4(\beta-1)}{2 \beta-1} e^{-C t}
\end{aligned}
$$

for some uniform positive constant $C$. If $t>\frac{R}{2}$, then

$$
\begin{aligned}
|\tilde{F}| & \leq \frac{4(\beta-1)}{2 \beta-1} \int_{\frac{R}{2}}^{t} e^{-2 s} d s \\
& \leq \frac{2(\beta-1)}{2 \beta-1} e^{-R} \\
& \leq \frac{2(\beta-1)}{2 \beta-1} e^{-2(R-t)} .
\end{aligned}
$$


We finish the proof of Proposition 3.7.

Now in order to complete the proof of Theorem 1.2, it suffices to show that the geodesics arising from the blowing up are all of finite length. Based on the arguments of Proposition 2.7, we only need to show that replacing a long line segment ( $\beta$-geodesic) by a suitable chosen geodesic does reduces total energy. Since $\frac{\partial u}{\partial \theta}$ decays exponentially, we can reduce the general case to the special one that $u$ is $\theta$-independent. The argument is similar to that in the proof of Proposition 2.7, so we only give a sketch. By Lemma 3.3, Proposition 3.5 and Proposition 3.7, the length $L\left(u_{n}(\mathcal{C})\right)$ of the image of $\mathcal{C}$ under $u_{n}$ and the energy $E\left(u_{n}, \mathcal{C}\right)$ of $u_{n}$ over $\mathcal{C}$ can be estimated as following

$$
L\left(u_{n}(\mathcal{C})\right)=\left|\frac{\partial \tilde{u}_{n}}{\partial t}\left(\frac{1}{2} R_{n}\right)\right| e^{\frac{2\left(\beta_{n}-1\right)}{2 \beta_{n}-1} \frac{R_{n}}{2}} R_{n}+O\left(e^{-C R_{n}}\right)
$$

and

$$
E\left(u_{n}, \mathcal{C}\right)=\left|\frac{\partial \tilde{u}_{n}}{\partial t}\left(\frac{1}{2} R_{n}\right)\right|^{2} e^{\frac{4\left(\beta_{n}-1\right)}{2 \beta_{n}-1} \frac{R_{n}}{2}} R_{n}+O\left(e^{-C R_{n}}\right)
$$

where $C$ is a uniform constant independent of $n$. If $L\left(u_{n}(\mathcal{C})\right)$ is tending to $\infty$ as $n \rightarrow \infty$, we replace the image $u_{n}(\mathcal{C})$ by a geodesic which is chosen to be in a suitable homotopy class, the total homotopy class of $u_{n}$ remains the same, and with much smaller length as in Case 1 and Case 2 in the proof of Proposition 2.7. Then we define a new map $u_{n}^{\prime}$ on $\mathcal{C}$ such that it is harmonic, maps $\mathcal{C}$ to the geodesic and parameterized with speed

$$
\left|\frac{\partial u_{n}^{\prime}}{\partial t}\right|=\left|\frac{\partial \tilde{u}_{n}}{\partial t}\left(\frac{1}{2} R_{n}\right)\right|
$$

The length of the geodesic is

$$
L\left(u_{n}^{\prime}(\mathcal{C})\right)=\left|\frac{\partial \tilde{u}_{n}}{\partial t}\left(\frac{1}{2} R_{n}\right)\right| S_{n}
$$

and the energy of $u_{n}^{\prime}$ on $\mathcal{C}$ is

$$
E\left(u_{n}^{\prime}, \mathcal{C}\right)=\left|\frac{\partial \tilde{u}_{n}}{\partial t}\left(\frac{1}{2} R_{n}\right)\right|^{2} S_{n}
$$

where $S_{n}$ is the length of $\mathcal{C}$ in the new parameterization and

$$
\left|\frac{\partial \tilde{u}_{n}}{\partial t}\left(\frac{1}{2} R\right)\right|\left(R_{n}-S_{n}\right)>C_{0}
$$

for some fixed constant $C_{0}>0$. We still use $u_{n}^{\prime}$ to denote the new maps obtained by replacing $u_{n}$ along $\mathcal{C}$ by $u_{n}^{\prime}$. Now it is easy to see that as $\beta_{n} \rightarrow 1$

$$
\begin{aligned}
E_{\beta_{n}}\left(u_{n}\right)-E_{\beta_{n}}\left(u_{n}^{\prime}\right) & \geq \frac{1}{2}\left(E\left(u_{n}\right)-E\left(u_{n}^{\prime}\right)\right) \\
& \geq \frac{1}{2}\left|\frac{\partial \tilde{u}_{n}}{\partial t}\left(\frac{1}{2} R\right)\right| C_{0}>0 .
\end{aligned}
$$

This contradicts to $u_{n}$ is $E_{\beta_{n}}$-energy minimizing in the fixed given homotopy class. Therefore we conclude that the length of the geodesic arising from every connecting cylinder is finite. The proof of Theorem 1.2 is now complete. 


\section{Application to minimal surfaces theory}

For any conformal structure $c$ on the compact Riemann surface $\Sigma$, there exists a minimizing harmonic nodal map $u_{c}$ into $M$ in any given homotopy class $a$ by Theorem 1.2. We set

$$
\alpha=\inf _{c \in \operatorname{Conf}(\Sigma)} E\left(u_{c}\right)
$$

where $\operatorname{Conf}(\Sigma)$ is the set of all conformal structures on $\Sigma$. Then let $u_{c_{n}}$ be a sequence of minimizing harmonic nodal maps in the homotopy class $a$, such that

$$
\lim _{n \rightarrow \infty} E\left(u_{c_{n}}\right)=\alpha
$$

and

$$
\lim _{n \rightarrow \infty} c_{n}=c_{\infty}
$$

where $c_{\infty}$ is a conformal structure on $\Sigma$ which may degenerate at finitely many points. According to Theorem 1.1, a subsequence of $u_{c_{n}}$ converges to a minimizing harmonic nodal map $u_{c_{\infty}}$. It is then well known and not hard to prove that $u_{c_{\infty}}$ is conformal with respect to $c_{\infty}$. Therefore, $u_{\infty}$ parameterizes a generalized minimal surface $\Sigma_{\infty}$ which is area minimizing in the homotopy class $a$.

On the other hand, we will show that the minimal surface $\Sigma_{\infty}$ is in fact admissible, i.e., if $B_{\infty}$ is the second fundamental form of $\Sigma_{\infty}$ then

$$
\int_{\Sigma_{\infty}}\left\|B_{\infty}\right\|^{2}<\infty
$$

A point $p \in \Sigma$ is called a cusp is there is a conformal map $F$ from the unit disk $D_{1}$ in $\mathbf{C}$ to a neighborhood $U$ of $p$ in $\Sigma$ such that the induced metric on $U$ is of the form

$$
F^{*} d s^{2}=|z|^{2 \alpha-2} g, \quad \alpha>1
$$

where $g$ is a regular Riemannian metric on $D_{1}$ and $z$ is a holomorphic coordinate on $D_{1}$.

We have the following result on the regularity of the minimal surfaces we just constructed.

Proposition 4.1. The minimal surface $\Sigma_{\infty}$ is admissible.

Proof. First, the minimal surface $\Sigma_{\infty}$ is a union of finitely many irreducible compact minimal surfaces. We only need to consider the cusp points. At such a point $p$, take any 2-dimensional component $\Sigma_{\infty}^{1}$ of $\Sigma_{\infty}$ which contains $p$. Since $f: \Sigma_{\infty}^{1} \rightarrow M$ is conformal,

$$
g=|\nabla f|^{2} g_{0}=4\left|\frac{\partial f}{\partial z}\right|^{2} g_{0}
$$


where $g_{0}$ is the regular metric on $D_{1}$. Since $f$ is also harmonic, we have near $p$ that

$$
\partial_{z} \partial_{\bar{z}} f=o\left(\left|\partial_{z} f\right|^{2}\right)
$$

Since $f$ is smooth, we can write

$$
\partial_{z} f=\sum_{p+q=l} c_{p \bar{q}} z^{p} \bar{z}^{q}+\text { higher order terms }
$$

where at least one coefficient $c_{p \bar{q}} \neq 0$ and $l \geq 1$. It then follows from the harmonic map equation

$$
\partial_{\bar{z}} \partial_{z} f=\sum_{p+q=l} q c_{p \bar{q}} z^{p} \bar{z}^{q-1}+\text { higher order terms. }
$$

If there is a $q$ bigger than 0 , then $\partial_{\bar{z}} \partial_{z} f$ is of order $l-1$. But $|\partial f|^{2}$ is of order at least $2 l$. Comparing to the harmonic map equation, we find this is impossible. Thus, the leading term of $\partial_{z} f$ is holomorphic. In other words,

$$
\partial_{z} f=c z^{l}+\text { higher order terms }
$$

for some non-zero constant $c$. Near each singular point where the curvature blows up, we construct a cut-off function as follows. Fix any large number $R>0$. Let $\phi$ be a smooth function from $[0, \infty)$ to $[0,1]$ such that

$$
\phi(t)= \begin{cases}1 & \text { if } t<R \\ 0 & \text { if } t>R+1\end{cases}
$$

and $\left|\phi^{\prime}(t)\right| \leq 1$ for all $t \geq 0$. We will use the cut-off function $\phi(\log (-\log r))$ where $r=|z|$. The curvatures in different metrics $g_{0}$ and $g$ are related by

$$
K_{g}=K_{g_{0}}-\partial_{z} \partial_{\bar{z}} \log \left|\partial_{z} f\right|^{2} .
$$

Then integration by parts leads to

$$
\begin{aligned}
& \left.\left|\int_{D_{1}} \phi(\log (-\log r)) \partial_{\bar{z}} \partial_{z} \log \right| \partial_{z} f\right|^{2} d A_{g}(z) \mid \\
\leq & \left.C_{1}\left|\int_{D_{1}} \phi(\log (-\log r)) \partial_{z} \partial_{\bar{z}} \log \right| \partial_{z} f\right|^{2} r^{2 l} d z d \bar{z} \mid \\
\leq & C_{2}\left(\left.\int_{D_{1}}\left(\frac{\left|\phi^{\prime}(\log (-\log r))\right|}{-r \log r}\right) r^{2 l}\left|\partial_{z} \log \right| \partial_{z} f\right|^{2} \mid\right. \\
& \left.+\left.\phi(\log (-\log r)) r^{2 l-1}\left|\partial_{z} \log \right| \partial_{z} f\right|^{2} \mid d z d \bar{z}\right)
\end{aligned}
$$




$$
\begin{aligned}
& \leq C_{3} \int_{e^{-e^{R+1}}}^{e^{-e^{R}}}\left(\frac{\left.\left|\partial_{z} \log \right| \partial_{z} f\right|^{2} \mid}{-r \log r} r^{2 l+1}+\left.\left|\partial_{z} \log \right| \partial_{z} f\right|^{2} \mid r^{2 l}\right) d r \\
& \leq C_{4} \int_{e^{-e^{R+1}}}^{e^{-e^{R}}}\left(\frac{1}{-r \log r} r^{2 l}+r^{2 l-1}\right) d r \\
& \leq C_{5} e^{-2 l e^{R}}\left(\log \left(-\log e^{-e^{R+1}}\right)-\log \left(-\log e^{-e^{R}}\right)+1\right) \\
& =2 C_{5} e^{-2 l e^{R}}
\end{aligned}
$$

where the constants $C_{1}, \ldots, C_{5}$ depend only on $g_{0}$ by choosing $R$ sufficiently large such that the leading terms dominate the rest terms in the power series expansion of $\partial_{z} f, \partial_{z} \partial_{\bar{z}} f$, and $\partial_{z} \partial_{\bar{z}} \log \left|\partial_{z} f\right|^{2}$. Therefore we have

$$
\lim _{R \rightarrow \infty} \int_{D_{1}} \phi(\log (-\log r)) \partial_{z} \partial_{\bar{z}} \log \left|\partial_{z} f\right|^{2} d A_{g}=0
$$

Hence if $p_{1}, \ldots, p_{s}$ are the cusps on $\Sigma_{\infty}$

$$
\left|\int_{\Sigma_{\infty}} K_{g} d A_{g}\right|=\left|\lim _{\epsilon \rightarrow 0} \int_{\Sigma_{\infty} \backslash \cup_{j=1}^{s} D_{\epsilon}\left(p_{j}\right)} K_{g} d A_{g}\right|<\infty .
$$

Since $\Sigma_{\infty}$ is minimal, it follows that

$$
\int_{\Sigma_{\infty}}\|B\|^{2} d A_{g}=-\int_{\Sigma_{\infty}} K_{g}<\infty
$$

which implies that $\Sigma_{\infty}$ is admissible.

Therefore we complete the proof of Theorem 1.3 on existence of minimal surfaces in a fixed homotopy class.

\section{Final remarks}

We first point out that the compactness result holds for boundary value problems as well. Let us only state a simplified version, namely we assume the image space is Euclidean. Therefore there are no bubbles. However, we need to take care of the long cylinders coming from blowing-up a neighborhood of degenerated points in $\Sigma_{\infty}$ as before. We shall state the result in a slightly different but equivalent fashion comparing to Theorem 1.1.

Given any compact Riemann surface $\Sigma$ with smooth boundary $\partial \Sigma$, we can embed $\Sigma$ into some Euclidean space $\mathbb{R}^{N}$. The boundary $\partial \Sigma$ is the union $\gamma_{1} \cup \ldots \cup \gamma_{k}$ of a collection of disjoint smoothly embedded curves $\gamma_{i}$ 's in $\mathbb{R}^{N}$. Let $\mathcal{S}_{i}$ be the 
set of diffeomorphisms from $\mathbb{S}^{1}$ onto $\gamma_{i}$. Set $\mathcal{S}=\left(\mathcal{S}_{1}, \ldots, \mathcal{S}_{k}\right)$. So $\mathcal{S}$ is the set of parameterizations of $\left\{\gamma_{1}, \ldots, \gamma_{k}\right\}$ by $k$ copies of $\mathbb{S}^{1}$.

Theorem 5.1. Let $\Sigma$ be a compact Riemann surface with smooth boundary $\partial \Sigma=$ $\gamma_{1} \cup \ldots \cup \gamma_{k}$. Take a sequence of parameterizations $\alpha_{i}=\left(\alpha_{i}^{1}, \ldots, \alpha_{i}^{k}\right) \in \mathcal{S}$ of $\partial \Sigma$ which converge to $\alpha_{\infty}=\left(\alpha_{\infty}^{1}, \ldots, \alpha_{\infty}^{k}\right) \in \mathcal{S}$. Let $\Sigma_{i}$ be $\Sigma$ equipped with the conformal structure $c_{i}$ which is determined by $\alpha_{i}$. Suppose that $\left\{f_{i}\right\}$ is a sequence of harmonic maps from $\left(\Sigma_{i}, \partial \Sigma_{i}\right)$ to $R^{N}$ satisfying: (1) $\left.f_{i}\right|_{\gamma_{j}}=\phi \circ \alpha_{i}^{j}$ where $\phi: \partial \Sigma \rightarrow R^{N}$ is smooth; (2) $f_{i}$ 's are in the same homotopy class relative to boundary. Then there is a subsequence of $\left\{f_{i}\right\}$ converges to a harmonic map $f_{\infty}$ from $\left(\Sigma_{\infty}, \partial \Sigma_{\infty}\right)$ to $R^{N}$ with $\left.f_{\infty}\right|_{\gamma_{j}}=\phi \circ \alpha_{\infty}^{j}$. Moreover, by adding finitely many geodesics $l_{j}$ 's of finite length, $f_{i}\left(\Sigma_{\infty}\right)$ converges to $f_{\infty}\left(\Sigma_{\infty}\right) \cup_{j} l_{j}$ in the relative homotopy class.

The phenomenon discussed in this paper can be generalized to higher dimensional manifolds. Instead of getting geodesics of finite length, the domain manifolds may collapse along spaces of 1-dimension less. Also, we believe the minimality in Theorem 1.1 can be weakened. We leave the further investigations to the interested readers.

\section{References}

[A] F.J. Almgren and W.K. Allard, On the radial behavior of minimal surfaces and the uniqueness of their tangent cones, Ann. of Math. 113 (1981), 215-265.

[CT] J. Cheeger and G. Tian, The cone structure of complete Ricci-flat manifolds with quadratic curvature decay, Invent. Math. (1994).

[Do] J. Douglas, Solution of the problem of Plateau, Trans. A.M.S. 33 (1931), 263-321.

[DT] W.-Y. Ding and G. Tian, Energy identity for a class of approximate harmonic maps from surfaces, Commu. in Analysis and Geometry Vol. 4(3) (1995), 543-554.

[EW] J. Eells and J.C. Wood, The existence and construction of certain harmonic maps, Symposia Math. Vol XXVI, 123-138, Academic Press, London-New York 1982.

[EW] A. Futaki, Non-existence of minimizing harmonic maps from 2-spheres, Proc. Japan Acad. 56 (1980), 291-293.

[Gr] M. Gromov, Pseudo holomorphic curves in symplectic manifolds, Invent. Math. 82 (1985), 307-347.

[GT] D. Gilbarg and N. Trudinger,Elliptic partial differential equations of second order, Grundlehren Math. Wiss. 224, Springer-Verlag, Berlin 1983.

[Gu] R. Gulliver, Regularity of minimizing surfaces of prescribed mean curvature, Ann. of Math. 97 (1973), 275-305.

[Hi] S. Hildbrandt, Boundary behavior of minimal surfaces, Arch. Rat. Mech. Anal. 35 (1969), 47-82.

[J1] J. Jost, Two-dimensional Geometric Variational Problems, John Wiley \& Sons, Chichester 1991.

[J2] J. Jost, Conformal mappings and the Plateau-Douglas problem in Riemannian manifolds, J. Reine Angew. Math. 359 (1985), 37-54.

[Mo] C.B. Morrey, Jr., The problem of Plateau in a Riemannian manifold, Ann. of Math. 49 (1948), 807-851.

[MY] W. Meeks and S.T. Yau, Topology of three dimensional manifolds and embedding 
problems in minimal surface theory, Ann. of Math. 112 (1980), 441-484.

[MSY1,2] W. Meeks, L. Simon and S.T. Yau, The existence of embedded minimal surfaces, exotic spheres, and positive Ricci curvature, Ann. of Math. 116 (1982), 221-259 and 621-659.

[P] T. Parker, Bubble tree convergence for harmonic maps, J. Diff. Geometry 44 (1996), 595-633.

[Q] J. Qing, On singularities of the heat flow for harmonic maps from surfaces into spheres, Comm. in Analysis and Geometry 3(2) (1995).

[QT] J. Qing and G. Tian, Bubbling of the heat flows for harmonic maps from surfaces, Comm. in Pure Appl. Math. 50(4) (1997), 295-310.

[Os] R. Osserman, A proof of regularity everywhere of the classical solution of Plateau's problem, Ann. of Math. 91 (1970).

[PW] T. Parker and J. Wolfson, A compactness theorem for Gromov's moduli space, J. Geom. Anal 3 (1993), 63-98.

[RT] Y.B. Ruan and G. Tian, A mathematical theory of quantum cohomology, Journal of Differential Geometry 42(2) (1995), 259-367.

[S1] L. Simon, Asymptotics for a class of nonlinear evolution equations with applications to geometric problems, Ann. of Math. 118 (1983), 525-571.

[S2] L. Simon, Isolated singularities of extrema of geometric variational problems, in: E. Giusti (ed.), Harmonic mappings and minimal immersions, Lecture Notes in Mathematics 1161, Springer-Verlag, Berlin-New York 1985.

[SU1] J. Sacks and K. Uhlenbeck, The existence of minimal immersions of 2 spheres, Ann. of Math. 113 (1981), 1-24.

[SU2] J. Sacks and K. Uhlenbeck, Minimal immersions of closed Riemann surfaces, Transc. Amer. Math. Soc. 271 (1982), 639-652.

[ScU] R. Schoen and K. Uhlenbeck, A regularity theory for harmonic maps, J. Diff. Geom. 17 (1982), 307-335.

[SY] R. Schoen and S.T. Yau, Existence of incompressible minimal surfaces and the topology of 3-manifolds with non-negative scalar curvature, Ann. of Math. 110 (1979), $127-142$

[TT] F. Tomi and A. Tromba, Existence theorems for minimal surfaces of non-zero genus spanning a contour, Mem. Amer. Math. Soc. 71 (1988).

[Ye] R.G. Ye, Gromov's compactness theorem for pseudo-holomorphic curves, Trans. Amer. Math. Soc. 342(2) (1994), 671-694.

[W] C.Y. Wang, Bubble phenomena of Palais-Smale-like sequences from surfaces to general targets, Houston J. Math. 22(3) (1996), 559-590.

Jingyi Chen and Gang Tian

Department of Mathematics

Massachusetts Institute of Technology

Cambridge, MA 02139

USA

(Received: 1 April 1997; revised: 15 April 1998) 